\title{
Topological Analysis of a Novel Compact Omnidirectional Three-Legged Robot with Parallel Hip Structures Regarding Locomotion Capability and Load Distribution
}

\author{
David Feller * ${ }^{(D)}$ and Christian Siemers
}

check for

updates

Citation: Feller, D.; Siemers, C. Topological Analysis of a Novel Compact Omnidirectional

Three-Legged Robot with Parallel Hip Structures Regarding Locomotion Capability and Load Distribution. Robotics 2021, 10, 117. https://doi.org/10.3390/ robotics10040117

Academic Editor: Donghun Kim

Received: 17 September 2021

Accepted: 27 October 2021

Published: 31 October 2021

Publisher's Note: MDPI stays neutral with regard to jurisdictional claims in published maps and institutional affiliations.

Copyright: (C) 2021 by the authors. Licensee MDPI, Basel, Switzerland. This article is an open access article distributed under the terms and conditions of the Creative Commons Attribution (CC BY) license (https:// creativecommons.org/licenses/by/ $4.0 /)$.
Institute of Electrical Information Technology, Clausthal University of Technology, D-38678 Clausthal-Zellerfeld, Germany; christian.siemers@tu-clausthal.de

* Correspondence: david.feller@tu-clausthal.de

\begin{abstract}
In this study, a novel design for a compact, lightweight, agile, omnidirectional three-legged robot involving legs with four degrees of freedom, utilizing an spherical parallel mechanism with an additional non-redundant central support joint for the robot hip structure is proposed. The general design and conceptual ideas for the robot are presented, targeting a close match of the well-known SLIP-model. CAD models, 3d-printed prototypes, and proof-of-concept multi-body simulations are shown, investigating the feasibility to employ a geometrically dense spherical parallel manipulator with completely spherically shaped shell-type parts for the highly force-loaded application in the legged robot hip mechanism. Furthermore, in this study, an analytic expression is derived, yielding the calculation of stress forces acting inside the linkage structures, by directly constructing the manipulator hip Jacobian inside the force domain.
\end{abstract}

Keywords: legged robot; locomotion; spherical parallel manipulator; kinetostatic analysis; multibody simulation

\section{Introduction}

Legged robots and their locomotion is an highly active field of research. Regarding the impressive ability of living beings to traverse even and complicated uneven terrain, researchers are motivated to understand and recreate natural locomotion with the development of walking robots. In this regard, remarkable results have been proposed in the past, getting robots to walk over difficult terrain without falling over or being able to stabilize themselves after loosing balance. While there exist a huge number of different legged robots, many conceptual differences in design, implementation, field of use, and control strategies are present. Hence, in this study, a new conceptual design for a legged robot that differs from often biologically inspired walking robots is proposed and analysed.

\subsection{The SLIP Model}

Considering natural and energy efficient locomotion, a well known mathematical concept describing the locomotion of animals is the spring-loaded inverted pendulum (SLIP). This SLIP model [1] was initially proposed by Blickhan [2], although prior work goes back to McMahon and Greene [3], proposing early concepts of the model. The SLIP model assumes the body mass concentrated in one point and a massless leg, comprising a linear spring, acting between body and ground contact, see Figure 1. The model is divided in two alternating phases, stance and flight. In the stance phase, the leg has ground contact and gets compressed, while in the flight/swing phase, the leg is fully expanded and has no ground contact. Often, the pure SLIP model is expanded by a hip joint for an additional leg anchored in the body centre for the purpose of bipedal locomotion and is analysed in just two dimensions for simplicity. With two legs being in contact, the model involves a double-stance phase, which is inherently necessary for the modelling of walking 
in addition to running that only considers one ground contact at a time. An important note is that the SLIP behaviour is not only achievable by the mechanical design but also by the implemented control algorithms [1], which can produce SLIP characteristics. With regard to energy efficient locomotion, the implementation of the springs in the SLIP design is of high significance, as they provide energy storage and therefore enable passive dynamics of the legged robot, lowering the requirements of energy consumption and necessary power of the actuators. Moreover, regarding a serial arrangement of the motor, the spring, and the leg joint, springs are important for impact reduction at the moment of foot-to-ground contact while walking, acting as a decoupling element [4] between the mechanical structure and the motor inertias.

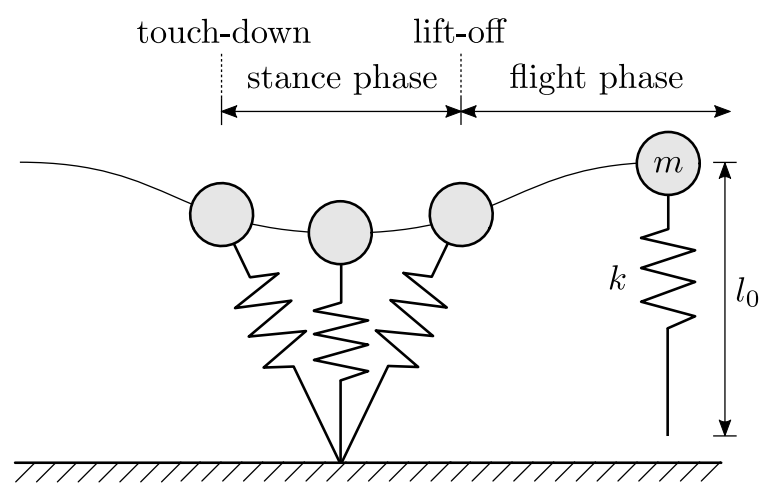

Figure 1. SLIP model for dynamically balanced running with body point mass $m$, spring stiffness $k$, and uncompressed leg length $l_{0}$.

Since this very simple model resembles the natural gaits and ground contact force profiles of humans and animals very accurately, it is researched actively and serves as a profound template for legged locomotion. Due to the successful application of this model considering walking of legged robots, as it was shown in simulation and with real robots in many publications, the SLIP model serves as a conceptual and fundamental target for the robot presented.

\subsection{SLIP-Based Real World Locomotion}

Since the SLIP model is an idealized model, a real robot is only able to accomplish this design to a certain degree due to mechanical design considerations. Hence, in contrast to the theoretical model, a real robot has legs with mass and inertia, which can not kinematically move instantaneously to a target position. Additionally, the total body mass is not reduced to a single concentrated point; therefore, inertia is present. With the transition into the real world with an actual mechanical robot model, the introduction of a third dimension will likely produce deviations from the idealized planar SLIP model, due to mechanical constraints of real world prototypes, as the body centre of mass and the individual hip joints will not be able to share the same location. An exception to a certain degree are one-legged hopping robots, which can match the SLIP model in three dimensions very closely. In this regard, notable famous examples of robots, amongst others, approximating the SLIP model closely are the well-known one-legged Raibert-hopper [5], the two-legged robot ATRIAS [6], and the RHex hexapod [7]. Considering the foot contacts, no perfect point contact will be possible, and contacts will obey a dynamic model, comprising the handling of stiction and sliding forces. Furthermore, the ideal SLIP model assumes energy conservation, while actual ground contacts involve energy dissipation.

\section{Methods}

In the following, first a conceptual robot design is derived and presented without the actual implementation of springs, serving as a proof of concept. The conceptual robot is considered only regarding the topology of the individual rigid bodies comprising the physical multi-body robot model. Hence, two possible robot topologies and their 
implications on the joint loads are compared, resulting in considerations for future design iterations and the general usability of the robot for legged locomotion. The emphasis in this study concerns the usability and the implication of the underlying mechanical structure, which is utilized to accomplish the overall targeted robot design and topology. Regarding the untypical robotic layout, this study aimed to answer the question to what extent the chosen mechanical design is suitable to enable legged locomotion.

For the analysis, the conceptual model is performing several motion profiles in simulation, including a basic walking sequence. The resulting load reaction forces and torques defined by the structure of the robot under motion and the required actuator torques were analysed, especially observing the individual joint loads of both robot topologies regarded in this examination. With the results obtained from this conceptual model, a final robot model including springs as a measure to add passive elasticities as a key element to approximate the SLIP model is presented and discussed briefly in the later sections of this proposal.

\subsection{Design and General Topology}

As stated above, the design for the robot depicted in this proposal tried to resemble the SLIP model as closely as possible. To accomplish this, the general design targets a lightweight and dynamically agile robot with the mass centred at the torso and with a low leg-to-torso weight ratio, approximating the SLIP model and incorporating a series arrangement of actuator and elasticities in the leg construction. The distribution of the robot mass was considered as the main focus on finding an optimal mechanical design, realizing low leg mass and inertias, reducing the required actuator power as much as possible, and utilizing light materials and 3d-printing for creating shapes that enable a highly compact construction and kinematically dextrous robot.

Assuming that the robot feet are small enough to be approximated as simple point contacts, at least three legs are necessary for statically stable standing, which was also chosen as the minimal number of legs for keeping the overall robot weight as low as possible. Moreover, by using three legs, the control strategy can be simplified into an hybrid control algorithm, enabling motions that fluently change between statically stable poses and partially dynamically balanced poses. By nature, a three-legged design leads immediately to a radial symmetrical layout. Therefore, the robot is capable of omnidirectional movement, and no primary orientation of the robot torso for walking in arbitrary directions is intended; although, due to non-circular symmetry of the robot to any axis, there may exist optimal orientations considering walking performance or energy efficiency. Many robots found in the literature are designed with biological examples in mind, yielding typically symmetrical bipedal, quadrupedal, or hexapedal robots. Noteworthy examples of other non-biologically based three-legged robots can be found at $[8,9]$, although they do not necessarily rely on the SLIP model. In this regard, the locomotion of the robot in [8] is based on leg-length variation, while the one in [9] performs a ful flip of its legs around its own torso. The robot presented in this study is considered to perform more natural leg motions but is composed of an uneven number of legs, which is in contrast to the typical biological counterpart. This raises the question about the sequence of stance and flight phases, since no clear symmetrical arrangement of the robot legs in respect to the walking direction is apparent unlike with most other robots.

Figure 2 shows the basic topological design concept of the robot presented. The robot is composed of a central torso, with three legs attached in radial symmetrical order. The angle between neighbouring legs equals $120^{\circ}$. Each leg comprises a spherical hip joint and an revolute knee joint, resulting in an $\underline{\mathrm{S}}$-type mechanism. In this article, underline notation depicts actuated joints, in contrast to passively connected joints. If all three legs are in contact with the ground, the robot can also be considered as an $3 \underline{S R S}$ parallel manipulator, enabling a six-degree-of-freedom motion of the robot torso. Since dynamically stable, fast, agile, and energy efficient locomotion is targeted, the following additions and design decisions for the internal mechanical design were made. 


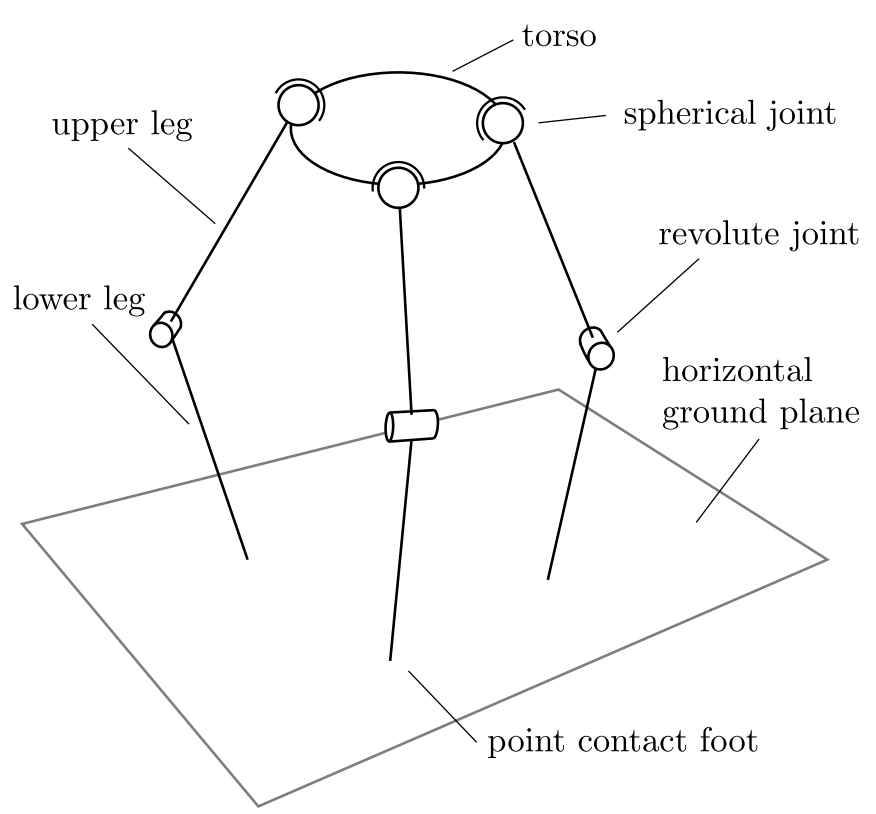

Figure 2. General conceptual robot design overview. The feet are considered as points, colliding with an even ground plane.

\subsection{Internal Mechanical Design}

By design, the robot legs are comprised of a one-degree-of-freedom (DOF) rotatory knee joint, and a spherical 3-DOF hip joint, leading to 4 DOF per leg and a total number of 12 DOF. Thus, if one only considers the foot position to be controlled, the leg mechanism is redundant, having one extra DOF, which may be useful regarding obstacle avoidance or locomotion performance. In the following sections, concepts for the internal mechanical actuation of these joints are evaluated.

Many robots use a serial concatenation of actuators for enabling multiple DOFs per joint, but this approach will likely increase the inertia of the leg and reduce the agility of the leg movements, since serial mechanisms have to cope with the mass and inertia of its following actuator and mechanical structures. By using a parallel arrangement of the actuators, they can be placed near the centre of the robot torso, concentrating the heavy components of the robot at a very narrow volume, which is mandatory to obtain the SLIPlike mechanical structure. Moreover, it is shown by ATRIAS, which uses a four-bar parallel mechanism [6] for its legs, that this design is useful at shifting the mass concentration to the upper body, resulting in very lightweight legs. In general, the benefits of a parallel layout are faster and more precise movements, compared to serial mechanisms, which is utilized, for example, in typical industrial Delta robots. Due to light linkage structures with low inertia and actuators, which are not part of the moving structure itself, high motion speed and acceleration capability is typical for these types of manipulators. Additionally, due to their parallel structure, which leads to high mechanical rigidity and load-capacity, these manipulators offer great motion accuracy. On the other side, parallel mechanisms also suffer from a smaller overall workspace than mechanisms with a serial design, since the reachable workspace is only the intersection of the individual workspaces of each linkage of the parallel structure [10]. Since this workspace limitation was considered as no significant drawback for the targeted operation in this study, the benefits outweigh it in this application; therefore, a parallel actuator layout was chosen.

As a consequence, the hip joint therefore utilizes the spherical parallel manipulator (SPM), which is studied in several publications, e.g., [11-14]. As a fast and accurate mechanism, this type of parallel manipulator is suitable for any general orientation task; therefore, it can be used as a device for orienting e.g., antennas, mirrors, lasers, solar panels, workpieces, and telescopes, as stated in [14,15]. In [14,16], a manipulator for precise and high-speed orientation of a camera-the Agile Eye-was built. In [17-19] the 
manipulator was utilized as an device for surgery applications and in [20] in a human hip exoskeleton context. In combination with the knee joint, each leg of the robot is a serial-parallel-manipulator on its own, which is connected by the central torso part.

\subsection{SPM-Based Walking}

Considering walking robots, the SPM appears to be utilized quite rarely. Examples of actual implementations are only found in $[21,22]$, which suggests that a deeper investigation on the possible suitability for the purpose of robot locomotion is of interest.

Since a parallel mechanical layout is chosen for the hip actuation, additional mechanical options exist for the placement of the knee actuators. For the conceptual robot, a simple design was chosen, placing the actuator inside the upper leg with axis symmetrical orientation and assuming an additional bevel gear connection for transmitting the actuator torque into the knee joint, as shown in Figure 3. The concept aims to optimize the mass distribution for reduced inertia effects by placing the three hip actuators inside the robot torso and the knee actuator as near as possible to the spherical hip joint. Since the overall mass of the robot is located inside the main body by design, this will reduce the necessity to cope with large masses and inertias hindering the robot to perform fast movements of its legs. A more sophisticated design for the real-world application is explored in the final robot design in the conclusion of this article.

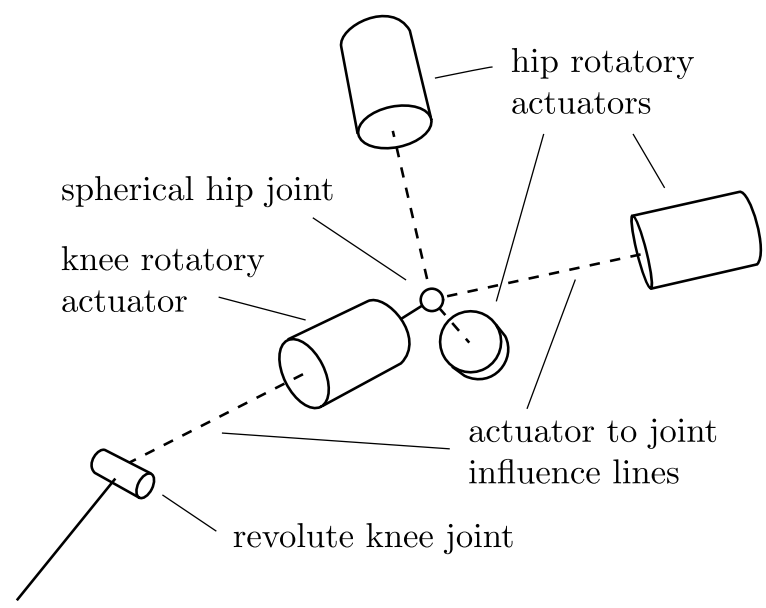

Figure 3. Actuator placement inside the leg. Dashed lines mark influence of the individual actuators on the actual topological joints of the robot model.

\subsection{The SPM as a Hip Joint}

In the following, the general concept, layout, benefits, and drawbacks of the SPM are discussed, and a real-world prototype model for the hip component is presented. A SPM is comprised of a base-platform and a tool-platform or end-effector, connected by three linkstructures in parallel order, each built by two links: a proximal and a distal link, as depicted in Figure 4. The links of the mechanism are connected with rotatory joints, while every of the nine-joint axes intersects in one common centre point of the manipulator. By actuating the three base joints, the tool platform can be rotated around this common axis intersection point, which also marks the location of the virtual spherical joint of the manipulator. This intersection point is also called the geometric centre, the centre of rotation or the origin of the manipulator in other publications, since all parts of the manipulator rotate around this particular location. The mechanism is also known as a 3RRR-parallel-manipulator or a 3-DOF SPM, because it has three rotatory degrees of freedom and three parallel linkages involving three revolute $(\mathrm{R})$ joints between the base-platform and the end-effector.

Since the orientation of the tool-platform is related to the individual motions of the parallel link structures, actuator torques and external load applied to the tool-platform have to be carried by the linkages. Considering that a small mechanical structure is needed for a low total mass of the robot and an overall compact design, a relatively high load will 
have to be exerted onto this mechanical structure. Without altering the general layout of the spherical parallel mechanism, as depicted in Figure 4, the resulting forces may bend and deform the linkage structure, leading to friction and impeded movements. Furthermore, as the individual linkages may be loaded with varying forces due to the manipulator being oriented in certain poses, which may produce highly unequally distributed external loads, mechanical manipulator rigidity is a central but also a critical aspect regarding a compact size of the system. In this regard, later sections in this article further investigate the load distribution onto the individual linkages.

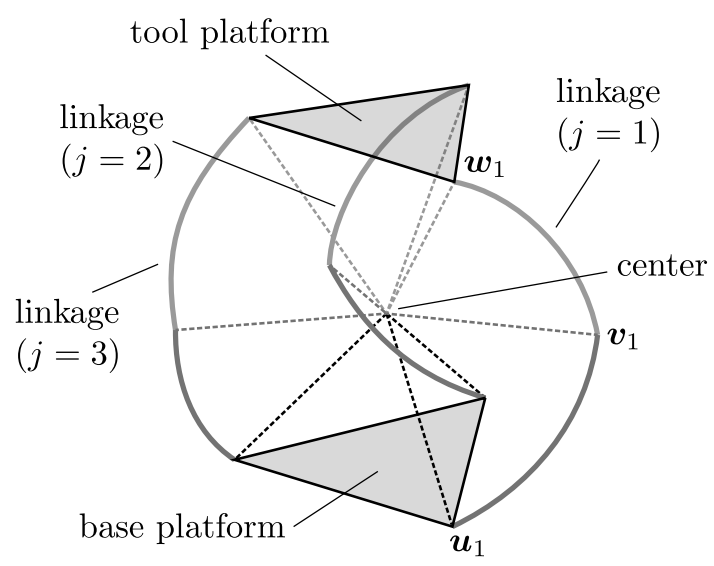

Figure 4. General layout of a spherical parallel manipulator. Dotted lines indicate actuated (black) and passive (gray) rotatory axes $\hat{u}_{j}, \hat{v}_{j}, \hat{w}_{j}$.

Implications of the performance of the SPM in the case of non-perfectly rigid links and the undesired translational shift of the tool-platform are regarded in [23]. The problem of having to cope with the static load of the robot itself and dynamic forces and torques exerted by moving the robot was also present in [22]. Here, a pin was used, loaded by a spring, applying a constant pressure on the tool platform. Since this method lacks capturing forces form arbitrary directions, a more robust attempt was employed in this work.

Another drawback of the SPM, as in general for parallel manipulators, is the smaller overall workspace due to the individual spatial dexterity of all involved parallel linkages. As detailed in the following, further workspace limitations of the manipulator are present due to the extended possibility of internal mechanical collisions, which may occur based on the altered mechanical design utilized in this study.

As discussed in the later material sections, due to the focus on compact design, traditional manufacturing processes could not be used for the complex geometric shapes of the parts, hence the parts are made by 3d-printing. With using resin-based printing methods, the durability, rigidity and stiffness of the resulting manipulator is also limited.

\subsection{Load Support Joint}

Since it is required to stress the individual linkages of the SPM as little as possible, regarding the mechanical rigidity that is achievable based on the compact design and the selected materials, in this study, an additional load-supporting joint was added to the hip joint mechanism, decoupling the external load from the SPM and capturing forces that otherwise would be exerted onto the linkage structures. By placing an actual spherical joint in the centre of the virtual rotatory centre, connecting the base- and tool-platforms, forces of arbitrary directions can be absorbed. Despite being mentioned in [23] and also commonly used with 3ㄹ-manipulators, an actual realized example of an 3RRR-manipulator with this centre joint was not found by the author.

Furthermore, the load support joint aims to fully decouple and capture vertical forces in parallel direction to the upper leg, normally applied to the tool-platform. Vertical forces will be applied to the load support joint without stressing the linkage structure of the SPM, but still allowing the transmission of torques form the SPM structure into the upper leg. 
Hence, the target of this addition is to use the actual SPM linkage just for supplying hip torques, and decouple as much as possible any external or self induced arbitrary directed forces applied to the hip joint from the torque transmitting linkage mechanism.

\subsection{Mechanical Redundancy and Topology}

At this point, manipulator redundancy problems have to be considered. The typical SPM is regarded as a $3 R R R$ manipulator, which actually is a mechanically redundant system [17]. This redundancy becomes obvious at realizing that each RRR linkage chain fully defines a spatial point that serves as a virtual centre for the rotatory motions performed by the linkage. Combining multiple chains in parallel results in an overconstrained respectively mechanically redundant system. This is problematic at a real-world manipulator, since the individual centre points may not perfectly align due to manufacturing errors of the parts. As it is stated in other publications [17,24], this redundancy can be mitigated by employing an $3 \underline{R C C}$ structure, but it can also lead to translation shift and therefore displacement errors.

Considering the desired additional decoupling and load-supporting joint, the baseplatform and the tool-platform directly define a spherical centre point, which again produces a redundant system with either the $3 \underline{R} R R$ or the $3 \underline{R} C C$ outer linkage structure. Hence, in this work and only regarding the simulation aspect of the manipulator, the linkage structure employed a $3 \underline{C} C C$ structure around the centre joint; therefore, the topological structure of the SPM was altered as depicted in Figure 5b, in comparison to the standard layout found in Figure 5a.
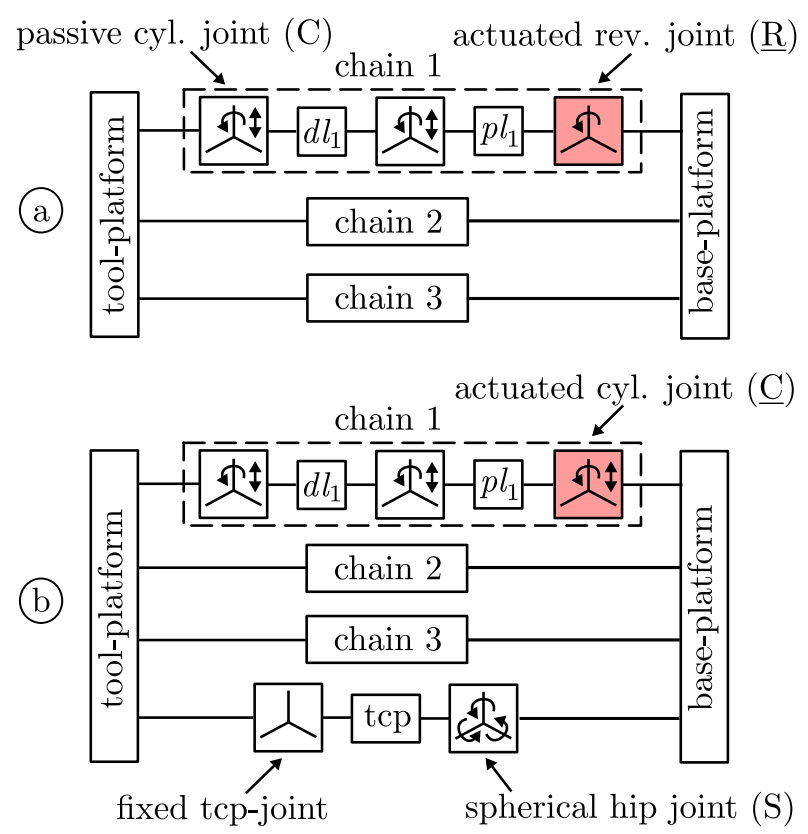

Figure 5. Typical $3 \underline{R C C}(\mathbf{a})$ and altered (b) topological non-redundant $3 \underline{C C C}+\mathrm{S}$ layout of the SPM. The topology of the single linkage structure with proximal link $p l$ and distal link $d l$ can be observed for both layouts. Red marks actuated joints. In the case (b), an additional joint with no DOF connects the tool-platform and an additional tcp body, which was included for load measurement purposes. Note that the tcp body in this model is the actual interface to external forces/torques or tools.

Employing the extended ([25], p. 13) Grübler's formula ([26], p. 243),

$$
F=6(l-n-1)+\sum_{i=1}^{n} f_{i}-f_{p}
$$

with the number $F$ for the resulting manipulator degrees of freedom, the number of links $l$, the number of joints $n$, the number of DOFs per joint $f_{i}$, and the number of passive DOFs 
$f_{p}$, it can be shown that a system with $F=3$ degrees of freedom is present for the nonoverconstrained topologies $3 \underline{R C C}$ without and $3 \underline{C C C}$ with spherical joint regarding Table 1. The $3 R R R$ configuration yields a negative $F=-3$, being mechanically overconstrained. An important note has to be made concerning the pure 3 CCC linkage structure without spherical centre joint, resulting in $F=6$, which implies that the parallel linkage structure in this configuration is actually unable to support any external forces at all; hence, it would drift apart without the spherical joint in its centre. This distinct property is important for the later load analysis of the manipulator. As a consequence of the robot containing closed kinematic loops, the identification of the robot structure being overconstrained or not is essential. Since the measurement of the joint reaction forces and torques in simulation is an important part of the further analysis, these measurements may fail at overconstrained systems, which can render some of the internal forces indeterminate ([27], p. 57). This behaviour is true also for the utilized SimMechanics toolbox, which has been tested by the author of this paper in this regard through the simulation of exemplary overconstrained systems, although the actual algorithm implementation of the SimMechanics toolbox used for the dynamics simulation is not specified by Mathworks.

Table 1. Configuration parameters for different topologies. Abbreviations for joint DOFs are base $(b)$, link $(l)$, tool $(t)$, hip $(h)$, knee $(k)$, foot-ground contact $(f)$, and passive $(p)$.

\begin{tabular}{ccccccccccc}
\hline Config. & $l$ & $n$ & $f_{b}$ & $f_{l}$ & $f_{t}$ & $f_{h}$ & $f_{k}$ & $f_{f}$ & $f_{p}$ & $F$ \\
\hline 3SRS & 8 & 9 & - & - & - & 3 & 1 & 3 & 3 & 6 \\
3RRR & 8 & 9 & 1 & 1 & 1 & - & - & - & 0 & -3 \\
3RCC & 8 & 9 & 1 & 2 & 2 & - & - & - & 0 & 3 \\
3CCC + S & 8 & 10 & 2 & 2 & 2 & 3 & - & - & 0 & 3 \\
3CCC & 8 & 9 & 2 & 2 & 2 & - & - & - & 0 & 6 \\
\hline
\end{tabular}

\subsection{Materials and Prototype}

Regarding the materials, the focus relied on combinations of 3d-printed plastic and aluminium parts for the use in mechanical assemblies. 3d-printing enables light and robust parts with highly complex shapes, while aluminium is used for highly stressed segments of the robot. The initial effort in building a prototype robot was already done and can be observed in the CAD model for the hip mechanism in Figure 16 and the corresponding realworld model in Figure 6a, showing a prototyped single hip joint of the robot. Resin parts were made of Formlabs White Resin V4 printed by a stereolithography process [28], which produces accurate parts needed for the precise assembly requirements. All linkage joints on the axes $\hat{\boldsymbol{u}}_{j}, \hat{\boldsymbol{v}}_{j}, \hat{\boldsymbol{w}}_{j}$ employ a miniature double-row angular contact ball bearing, hence preventing mechanical play of the joints, compared to typical ball bearings or bushings. Thus, any unwanted displacement of the individual part centre points from the common centre of the SPM is prevented by the specific selection of contact ball bearings, which is also shown in Figure $7 \mathrm{~b}$.

The spherical load support joint was realized by a ball and socket connection, utilizing an actual ceramic artificial hip joint, which is normally used for implantation in the human hip in the surgical procedure called arthroplasty. The ceramic joint and its snap-connector integration into the mechanism is depicted in detail in Figure 7a. As can be seen in the figure, the load support joint requires a connection to the base-platform, hence this obstructs the rotation of the proximal links and has to be considered in the mechanical linkage design. In regard to [14], the proximal links of the manipulator are constructed by two arc-shaped segments, which is necessary to avoid internal collisions with neighbouring proximal links. Due to the compact size of the manipulator in this work, in contrast to the $90^{\circ}$-angle of each arc segment as chosen in [14], the first $\left(\alpha_{u s}\right)$ and second $\left(\alpha_{s v}\right)$ part of the proximal links both feature an $54,74^{\circ}$-wide arc. The reduced angles of the sub arcs are required to avoid mechanical collisions of the proximal links with the upper leg part as they would interfere with the space required for the further leg construction at the top of the tool-platform. It should be noted here that, in general, it is possible to use a shape for the proximal link, 
comprising a single but curved arc, changing its radius over the distance from the base joint to the link joint, as seen, e.g., in [24]. Regarding the discussed prototype, this arc shape is not entirely possible due to the required avoidance of collisions with the central load support structure, which is the reason for the chosen mixture of the proximal linkage design. Regarding the optimal design [11] of an SPM, proximal and distal links still feature a resulting angle $\left(\alpha_{u v}, \alpha_{v w}\right)$ of $90^{\circ}$ between the actual rotatory axes $\hat{u}, \hat{v}$, and $\hat{w}$. The naming of the axes was done in alphabetical order of the axis from the start to the end of each linkage chain, which is different here from the naming convention found in most other publications. For reference, Table 2 displays the individual angles between different axes, as noted in Figure $7 \mathrm{a}$.

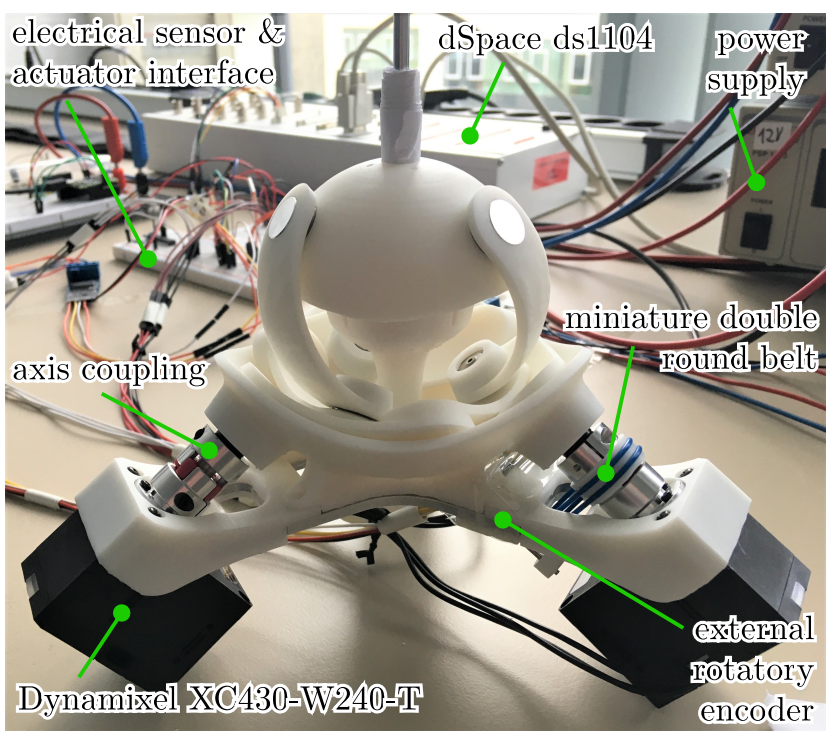

(a)

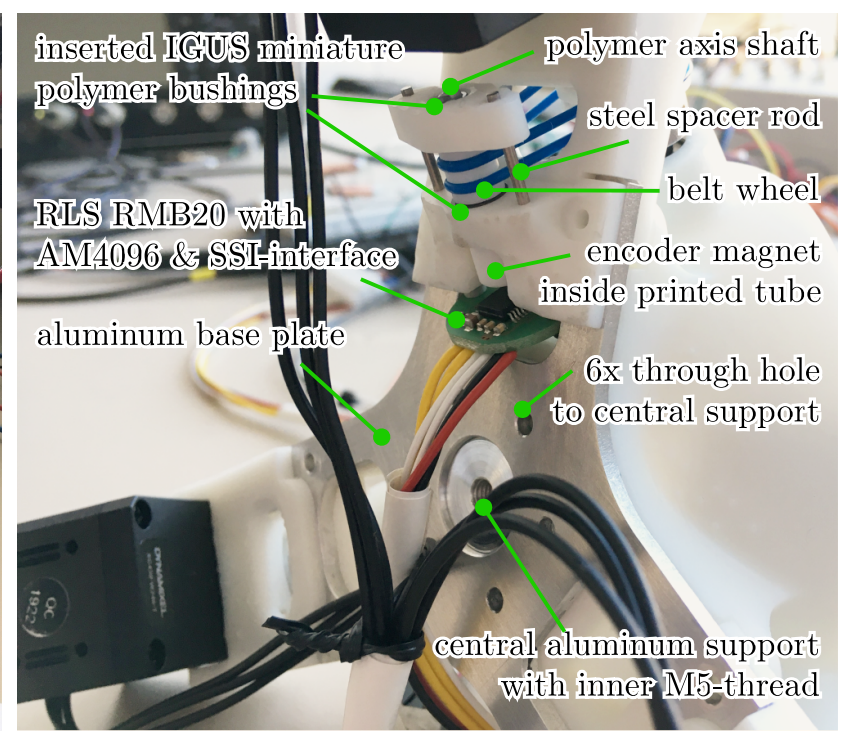

(b)

Figure 6. Real-world prototype (a) of the hip joint mechanism based on stereolithography 3d printing and thin supporting aluminium structures. Encoder integration (b) and visible screw holes for the torso connector structure on the bottom side of the manipulator unit.

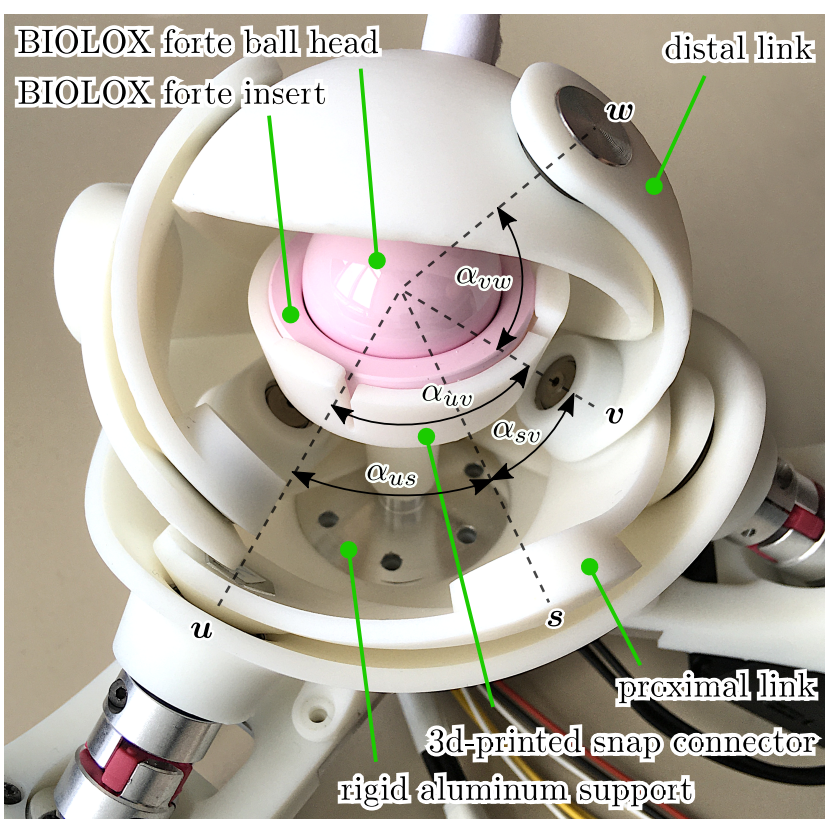

(a)

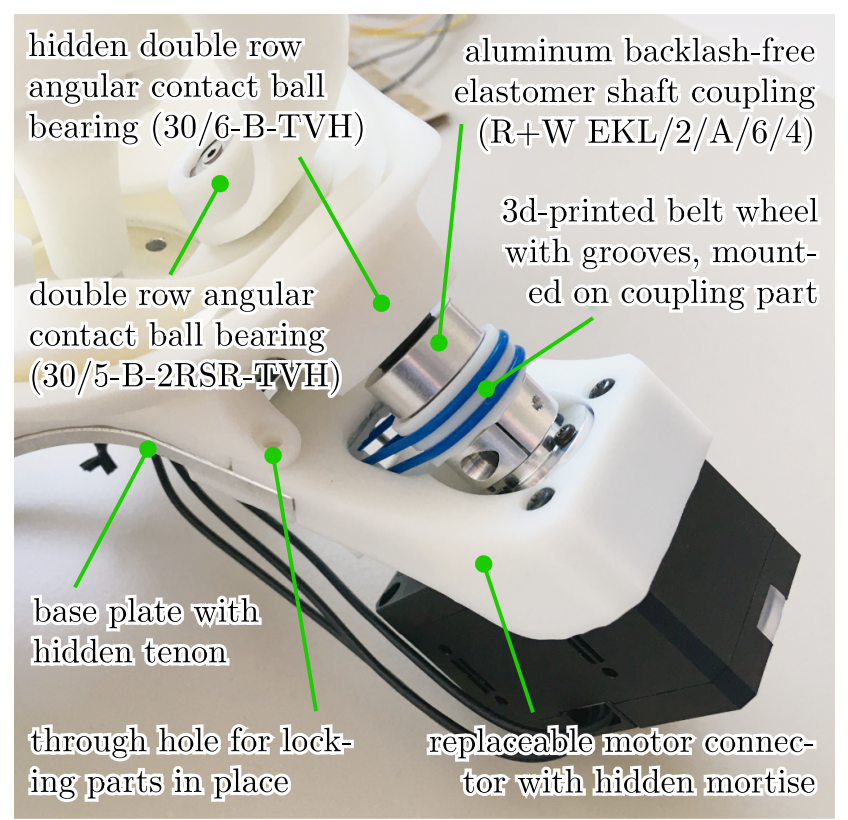

(b)

Figure 7. Spherical ball and socket artificial hip joint (a) made of ceramic by CeramTec [29]. Image (b) shows mechanical details of the motor connection. 
Table 2. Angles between intersecting axes of the SPM.

\begin{tabular}{lcccccc}
\hline Parameter & $\alpha_{u v}$ & $\alpha_{v w}$ & $\alpha_{u s}$ & $\alpha_{s v}$ & $\alpha_{u u}$ & $\alpha_{w w}$ \\
\hline Angle (deg) & 90 & 90 & 54.74 & 54.74 & 90 & 90 \\
\hline
\end{tabular}

Due to the selected materials and fabrication methods, it is possible to keep the overall weight of the robot skeleton relatively low, shifting the problematic aspect of the actual robot weight towards the actuators. With the weight of typical electrical DC- or BLDC/ECactuators with additional gearboxes in mind, an trade-off between the power and the weight of the actuators has to be made. For testing purposes, Dynamixel actuators of type XC430-W240-T [30] were used in this prototype, which will be replaced by better-suited custom actuators in future iterations of the robot. The actuators are held in place by a replaceable 3d-printed connector part that is fitted onto the aluminium base plate via a tendon and mortise connector, as shown in Figure $7 \mathrm{~b}$. A through hole connects and fixates the base plate, the base-platform, and the motor connector. The proximal links are connected via backlash-free shaft couplings to the actuators. The aluminium axis shaft and $3 \mathrm{~d}$-printed shape of the proximal links are fitted together via a hexagonal shaped press fitting, thus preventing unintended twisting between these parts. A contactless separate absolute rotatory encoder-IC of 12-bit resolution with an SSI-interface, decoupling the angular measurement objective from the actuator unit, was placed inside a gap in the base plate, below the mechanism, as depicted in Figure 6b. The encoder magnet axis rotates parallel to the proximal link axis via the employed miniature double-round belt. Due to the wheel ratio of $19 / 7$, the angular resolution was increased on the encoder side.

\section{Manufacturing and Mounting}

Since the $3 R R R$ mechanism is mechanically limited to spherical motion, extremely high manufacturing quality for meeting sufficiently low tolerances would be required to enable perfectly aligned motions of the individual linkage structures around the centre point of the mechanism. As done in the simulation by applying the CCC linkage structure to overcome the redundancy problem, using cylindrical joints enables the parts to self adjust for better alignment of the rotatory axis with the centre of the mechanism. Regarding rigid materials, this adjustable distance to the centre point is important for high motion performance. However, since the real-world parts are not perfectly rigid, compliance especially introduced by the resin parts exists, allowing the mechanism to be moving without blocking even with pure revolute joints; hence, the SPM linkage of the build prototype actually employs the overconstrained $3 \underline{R} R R$ structure. More rigid linkage parts, e.g., parts made of aluminium, would possibly require a reevaluation of the redundancy situation, especially since the manipulator discussed is of compact size, therefore only allowing minor inaccuracies. This will be evaluated in future revisions of the robot prototype.

Since assembling spherical parallel manipulators is often considered difficult, the parts for this prototype are shaped to not require the deformation of the links for the purpose of mounting the system. By first connecting the tool-platform and distal links, and then the base-platform and proximal links, both assemblies can be connected by orienting all links in a way that the link joint has its axis orientated horizontal, while vertically sliding the assemblies into each other. At last, a pin is inserted from the outside to connect the proximal and the distal link and is fixed by a screw from the inside of the manipulator, which can be seen in Figure 7a. Any deformation of the links still necessary only stems from small manufacturing errors, which are inevitably exposed at the mounting of overconstrained mechanical systems.

As a side note, considering a theoretical construction of an actual highly rigid 3RRR linkage, the decoupling of the load between the tool-platform and the hip support joint has to be revised. Regarding this specific scenario, the dedicated realization of a prismatic tcp joint between the tool-platform and the upper leg is necessary, as this joint will decouple the tool-platform from vertical forces but allow for the transmission of torque from the 
tool-platform onto the upper leg part. This design was actually considered in an early phase of this work but has not been considered furthermore due to the indeterminate nature of the resulting mechanical system.

\subsection{Legged Conceptual Prototype Model}

For the conceptual model, the above-described SPM model was used in circular arrangement to define the robot hip joints. By rotating the individual spherical parallel mechanisms around their local vertical axis, they can be arranged much closer in an interlocking manner, thereby further concentrating the overall robot mass on one point in the torso body. One actuator of each SPM unit fits between two actuators of the neighbouring units. This interlocking arrangement for the optimized distribution of masses in focus of the SLIP model is shown in Figure 8.

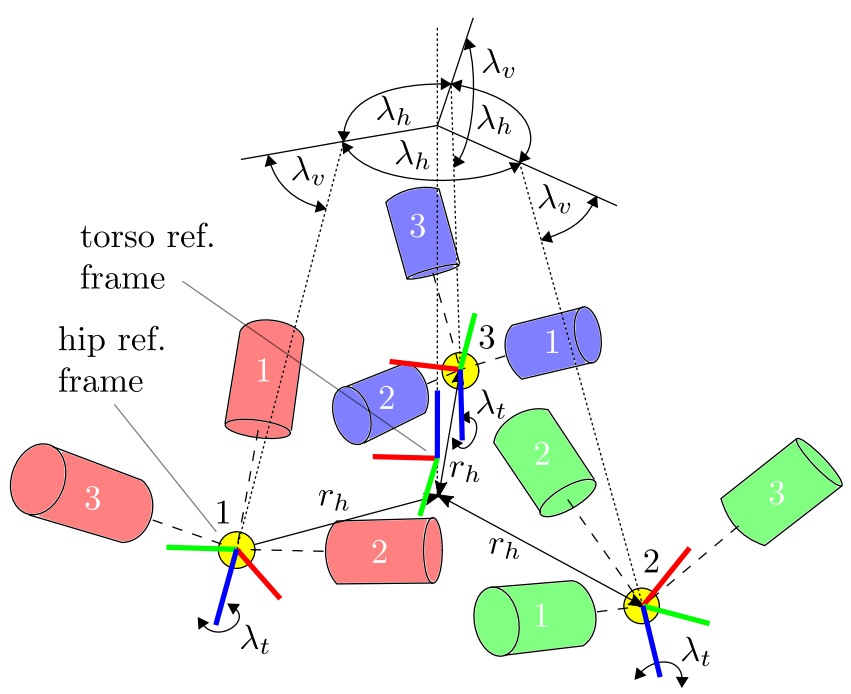

Figure 8. Interlocking radial symmetrical arrangement of the hip units around the vertical centre axis. Yellow circles mark the centre of the SPM units, and origins of the SPM centre reference frames. Red, green, and blue colours indicate $x-, y-$, and $z$-coordinates and individual hip units, respectively. White numbers relate to the individual branches, while black numbers identify the unit itself. The radius $r_{h}$ to the spherical centre of each hip joint is perpendicular to the vertical centre axis, which is the $z$-axis of the torso reference frame. SPM (3) is placed inside the $y$-z-plane of the torso reference frame.

The vertical angle $\lambda_{v}$, the horizontal angle $\lambda_{h}$, and the twist angle $\lambda_{t}$ were chosen by mechanical considerations, placing the units as close as possible without producing collisions but also allowing for a suitable workspace targeting legged locomotion. Iterative testing of different configurations in simulation while performing motions yielded the final design. It holds $\lambda_{v}=60^{\circ}, \lambda_{h}=120^{\circ}$, and $\lambda_{t}=5^{\circ}$. Table 3 expresses the orientation of the individual centre reference frames of the SPMs in relation to the orientation of the torso reference frame in Euler angles. Since the model features cuboid-shaped actuators, the packing density of the masses is limited, resulting in the smallest possible radius $r_{h}=0.1 \mathrm{~m}$. For the torso, a connector structure was employed, fixing the overall arrangement. The corresponding final CAD model of the assembly is depicted in Figure 9 and resembles the notation from Figure 8.

The remaining parts for constructing a full robot model are simplified shapes, which serve for the simulation of the conceptual model but lack the actual design necessary for operating in the real world. Therefore, upper-leg and lower-leg segments were assumed as cylindrical parts, while the knee actuator is located in the top of the upper leg. The masses and inertias of all parts were derived from the corresponding CAD models. Table 4 shows the physical properties, with the robot total mass $M \approx 2.05 \mathrm{~kg}$. Figure 10 shows the $3 \mathrm{~d}$ model of the robot in the simulation environment. Table 5 displays the geometric dimensions of the robot. With the SPM actuators having a mass of $44 \mathrm{~g}$ each, and assuming 
a mass of $100 \mathrm{~g}$ for the knee actuator, the actuators make up for approximately $34 \%$ of the total robot mass.

Table 3. Z-X-Z-Euler angles in degree of the individual hip centre frames in relation to the torso body reference frame.

\begin{tabular}{cccc}
\hline Hip $j$ & $Z_{0}$ & $X_{0}$ & $Z_{1}$ \\
\hline 1 & 120 & 150 & 5 \\
2 & -120 & 150 & 5 \\
3 & 0 & 150 & 5 \\
\hline
\end{tabular}

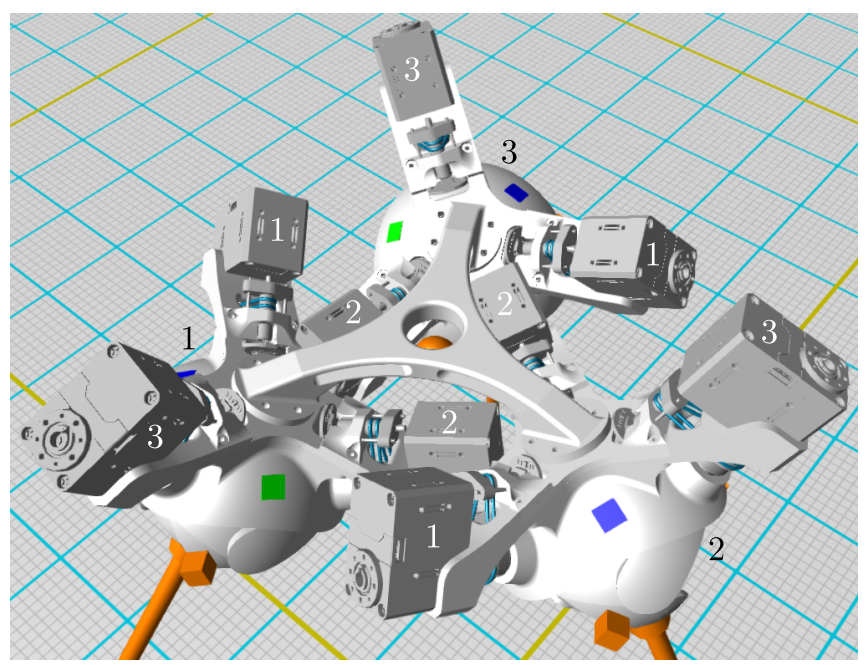

Figure 9. Robot assembly with actual CAD models, showing the interlocking arrangement of the individual hip units.

Table 4. Properties of the physical robot model. $N$ refers to the number of repeated assemblies in the full robot with part mass $m$.

\begin{tabular}{llrr}
\hline Assembly & $N$ & Mass (g) & $N \frac{m}{M}(\%)$ \\
\hline Torso-connector-structure & 1 & 47.504 & 2.32 \\
SPM base-platform & 3 & 354.199 & 51.84 \\
SPM prox. link & 9 & 27.810 & 12.21 \\
SPM dist. link & 9 & 10.009 & 4.39 \\
SPM tool-platform & 3 & 28.701 & 4.20 \\
Upper leg & 3 & 155.034 & 22.69 \\
Lower leg & 3 & 15.984 & 2.34 \\
\hline Robot & 1 & 2049.629 & 100.00 \\
\hline
\end{tabular}

\subsection{Simulation Framework and Setup}

In the following sections, a physical simulation of the conceptual model is carried out in Simulink, employing the Mathworks SimMechanics toolbox [31] for the internal mechanical representation of the robot model. For the construction, simulation, visualization, and reviewing, a specifically purposed simulation framework was built that will be further elaborated in later proposals, although screenshots and videos of the robot performing motions inside this framework are available at [32]. An overview is given in Figure 11, showing both the simulation components of the framework and the included hardware implementation. Both operating modes are controlled and visualized by an connected interface software. 
Table 5. Geometrical properties of the robot model.

\begin{tabular}{lcc}
\hline Parameter & Value & Unit \\
\hline Hip radius $r_{h}$ & 0.100 & $\mathrm{~m}$ \\
Foot radius $r_{f}$ & 0.240 & $\mathrm{~m}$ \\
Torso height $h_{t}$ & 0.510 & $\mathrm{~m}$ \\
Upper leg length $l_{u l}$ & 0.200 & $\mathrm{~m}$ \\
Lower leg length $l_{l l}$ & 0.300 & $\mathrm{~m}$ \\
\hline
\end{tabular}

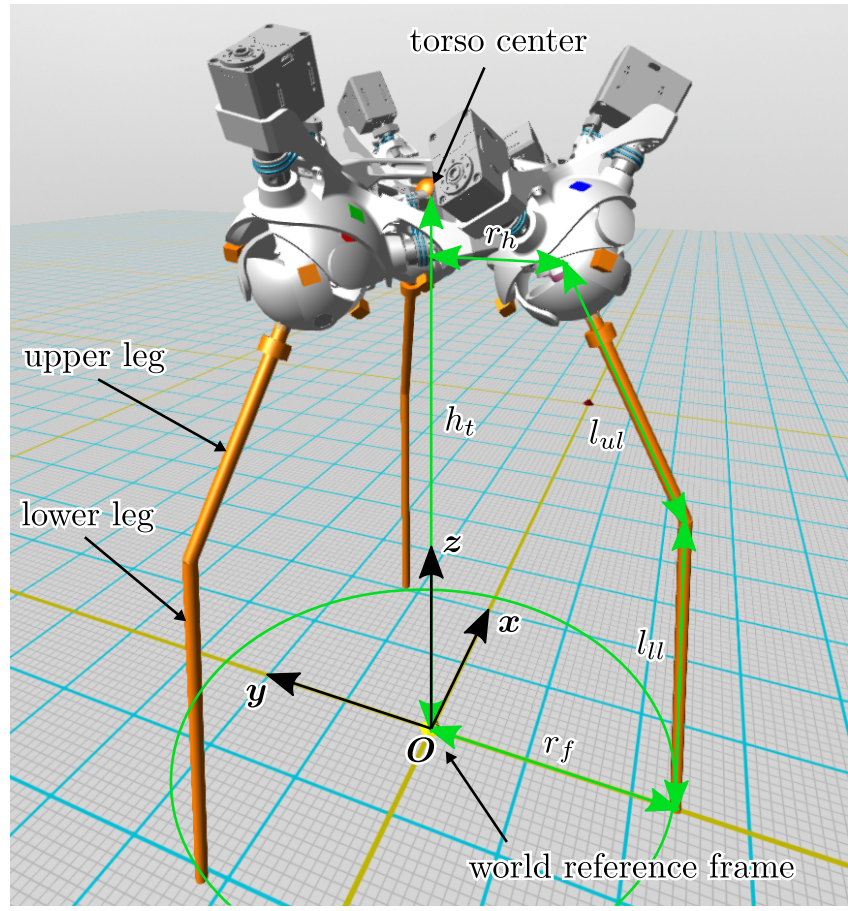

Figure 10. CAD model of the robot in the simulation framework. Grid distances and colour mapping: $1 \mathrm{~m}$ (yellow), $1 \mathrm{dm}$ (cyan), $1 \mathrm{~cm}$ (grey).

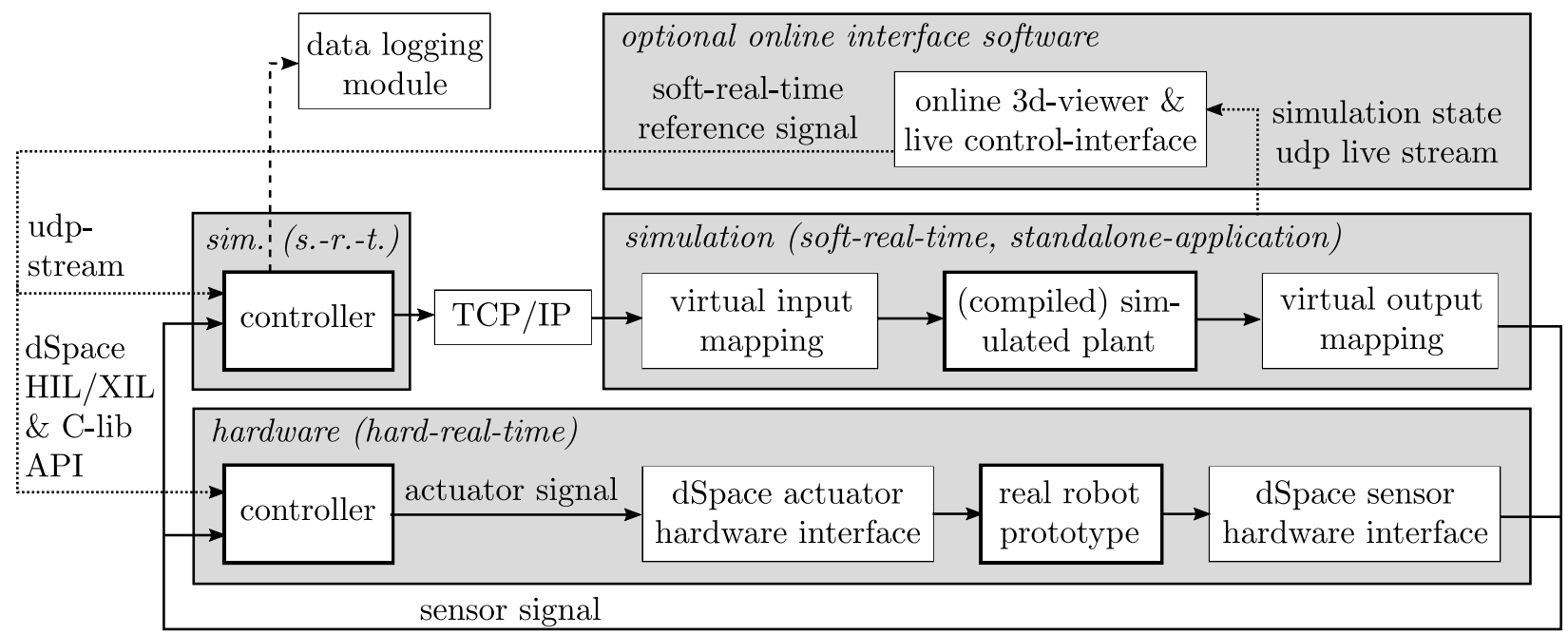

Figure 11. Simplified component view of the simulation framework. 


\subsubsection{Plant Model}

Figure 12 shows the plant model, including the mechanical system and also a specifically modelled behaviour for foot-ground contacts, joint friction, and joint limits. Specifically, the SimMechanics toolbox was used inside the plant model only for the computation of the direct dynamics of the multi-body system; any other mechanical behaviour was included manually. Detailed elaborations of the inner workings of the simulation are beyond the scope of this proposal, therefore only a brief overview is given.

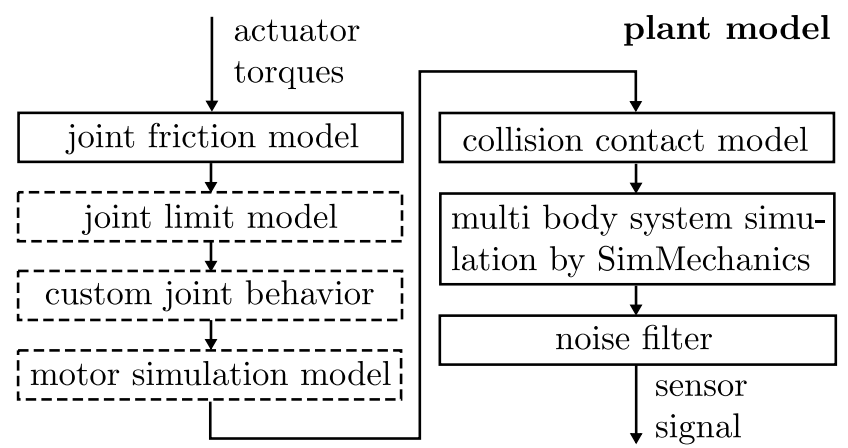

Figure 12. Overview of the plant model. Dotted parts were not active for the current robot model.

Ground contacts were implemented as linear laws for different cases by combining a surface-normal and surface-tangential reaction force. The normal force $f_{n}$ was calculated as the Kelvin-Voigt model ([33], p. 28), and the tangential force $f_{t}$ was modelled similar to [34] as a breakable spring damper system, which breaks at the transition from static to dynamic friction, yielding sliding, which is only based on velocity-dependent remaining forces. Static and dynamic friction forces are furthermore limited by the corresponding friction coefficients used in a dry Coulomb friction model. Hence, it was implemented with the penetration depth $p_{n}$ and the surface normal foot velocity $v_{n}$, which are both negative at increasing penetration,

$$
f_{n}= \begin{cases}\max \left(-k_{f} \cdot p_{n}-d_{f} \cdot v_{n}, 0\right) & \text { if } p_{n}<0 \\ 0 & \text { otherwise }\end{cases}
$$

for the surface normal reaction force. By the addition of only allowing for positive forces, ground sticking effects are avoided. With the tangential components of the foot position $p$ and the initial contact point $c$, the spring and damping parts of the tangential ground reaction force are defined,

$$
f_{t k}=-k_{f} \cdot\left(p_{t}-c_{t}\right) \text { and } f_{t d}=-d_{f} \cdot v_{t}
$$

which is then combined to

$$
f_{t}= \begin{cases}f_{t d} & \text { if }\left|f_{t k}+f_{t d}\right|>\left|f_{t, \max }\right| \\ f_{t k}+f_{t d} & \text { otherwise }\end{cases}
$$

The limiting maximum force is calculated by

$$
f_{t, \max }=f_{n} \cdot\left\{\begin{array}{ll}
\mu_{s} & \text { if }\left|v_{t}\right|<\varepsilon \\
\mu_{d} & \text { otherwise }
\end{array} .\right.
$$

Vector notation is omitted here but is included in the implementation, since the tangential reaction force depends on the velocity direction at foot-to-ground impact.

Since the SimMechanics toolbox does not include any form of energy dissipation inside the multi-body simulation, joint friction was included as an important aspect to achieve realistic behaviour of the model. Thus, friction was included as a Coulomb friction model, 
which depends on the computed internal joint reaction forces, orthogonal to the joint axis, combined with an velocity dependent component. The parameters were chosen by empirical tests, letting the model swing and come to rest without any actuator interference, resulting in similar behaviour to the real-world prototype. Distinctive experimentation is necessary in the future to achieve a more accurate match between the simulation and the real robot. Assuming that the joint axis is parallel to the local reference frame $z$-axis, the implementation reads with the current joint rotational velocity $\omega$,

$$
\tau_{\mu}= \begin{cases}-\operatorname{sgn}(\omega)\left(\mu_{j} \sqrt{f_{R, x}^{2}+f_{R, y}^{2}}+|\omega| \cdot d_{j}\right) & \text { if }|\omega|>0 \\ 0 & \text { otherwise }\end{cases}
$$

for the friction torque.

Mechanical joint limits were modelled by linear respectively rotatory spring-dampersystems, acting if a certain linear or angular threshold is passed, similar to the Kevin-Voigt model employed at the contact handling. For this analysis, joint limits were not enabled, as this would require the addition of $3 \mathrm{~d}$ collision tests based on the CAD models and would potentially invalidate the force/torque analysis concerning the joint loads.

Since the plant model runs at an integration step size much smaller than the controller system or the sensory devices attached to the model, a noise filter was added at last to smooth jittery signals and to reduce the possibility of capturing momentary numeric spikes inside the measurements at the moment of collision impacts, sending misrepresentative values to the controller model, running at a much lower frequency.

\subsubsection{Controller Model}

The controller depicted in Figure 13 includes a trajectory controller, calculating the target pose of the robot, and a full inverse kinematics solver for the model, calculating all required joint coordinates for any desired pose of the robot model. Despite the fact that the individual SPM mechanisms contain Dynamixel actuators with integrated controllers, a simplified positional PID-controller running at $1 \mathrm{kHz}$ was utilized for simulation purposes, since the robot was constructed as a conceptual design study and does not require an precise depiction of the actual behaviour of the Dynamixel actuators itself.

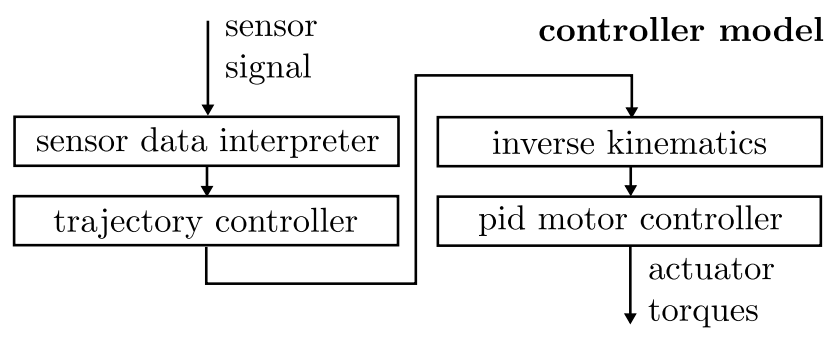

Figure 13. Overview of the controller model.

\subsubsection{Simulation Setup}

Considering the trade-off between the simulation accuracy, the numerical stability, and the execution speed of the compiled plant model, the Simulink solver ode2 (Heun) at a fixed time step of $\Delta t=2.5 \times 10^{-5} \mathrm{~s}$ for the mechanical system was chosen, which was selected as the fastest configuration possible with robust and stable behaviour for the robot model. Larger step sizes for the numerical integration showed unstable behaviour occurring at the moment of collision impacts, caused by the sudden penetration of the foots into the ground plane, resulting in large peaks of ground reaction forces. Hence, for this particular model, a small integration step size is required without the alternative of using softer contacts with reduced stiffness and damping parameters, which helps to lower impact forces at larger step sizes but also restricts the simulation accuracy. Thus, the parameters were chosen to keep the deviation between the behaviour of the robot model 
inside the simulation and its assumed behaviour in the real-world small. Table 6 sums up important simulation parameters.

Table 6. Simulation parameters of the plant model.

\begin{tabular}{lcc}
\hline Parameter & Value & Unit \\
\hline Floor stiffn. $k_{f}$ & 5000 & $\mathrm{~N} / \mathrm{m}$ \\
Floor damp. $d_{f}$ & 20 & $\mathrm{Ns} / \mathrm{m}$ \\
Static fric. $\mu_{s}$ & 0.8 & - \\
Dyn. fric. $\mu_{d}$ & 0.7 & - \\
Velocity threshold $\varepsilon$ & $1.0 \times 10^{-3}$ & $\mathrm{~m} / \mathrm{s}$ \\
Plant step time $\Delta t$ & $2.5 \times 10^{-5}$ & $\mathrm{~s}$ \\
Contr. step time $\Delta t$ & $1.0 \times 10^{-3}$ & $\mathrm{~s}$ \\
Simulink solver & ode $2(\mathrm{Heun})$ & - \\
Joint fric. coeff. $\mu_{j}$ & 0.01 & $\mathrm{Nm} \cdot \mathrm{s} / \mathrm{rad}$ \\
Joint fric. damp. $d_{j}$ & $10^{-4}$ & \\
\hline
\end{tabular}

\subsection{Motion, Load and Actuation Analysis}

Since the influence of the additional load support joint onto the robot model is of interest, all motions were performed with an identical reference trajectory for both possible robot topologies $T_{\triangle}$ without and $T_{\boldsymbol{\Delta}}$ with a load support joint, as shown in Figure 14. Both topologies represent a structurally (positions and orientations of bodies and centre of masses), kinematically (motion capabilities), and physically (masses and inertias) identical robot model, with the only difference being the internal joint connections. Therefore, a direct comparison between these configurations was possible and was employed to reveal the benefits and drawbacks of the additional load support joint.

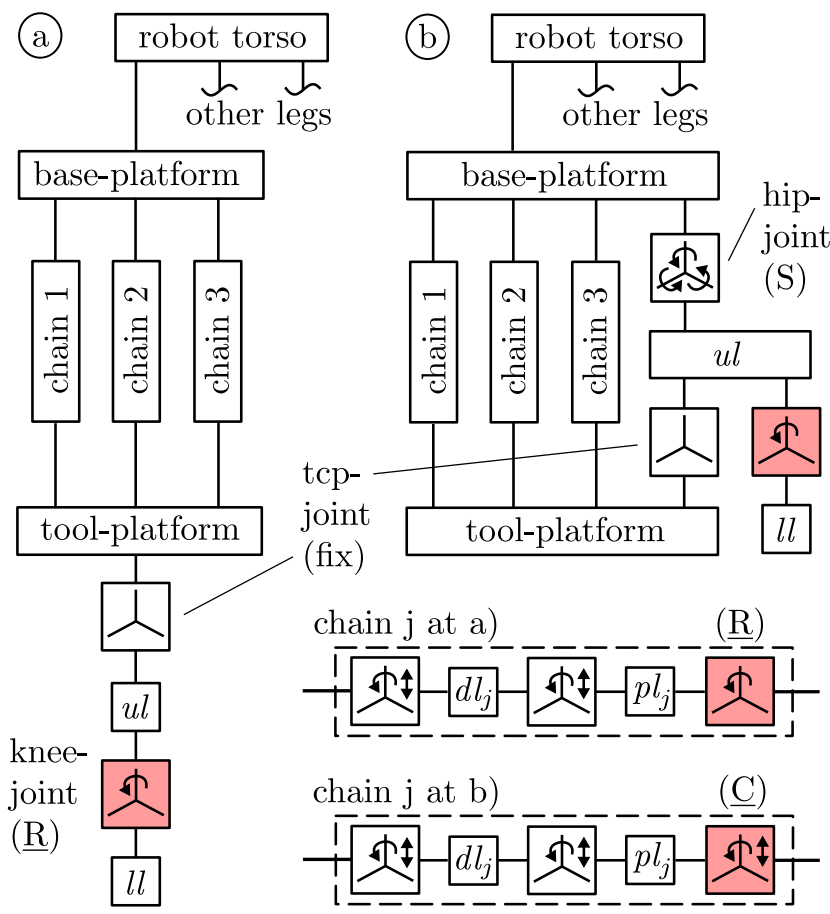

Figure 14. Full robot topology $T_{\triangle}$ without (a) and topology $T_{\Delta}$ with supporting load joint (b). Red-coloured connections are actuated (active) joints. Other joints are passive, non-actuated joints. Abbreviations are $p l$ for proximal link, $d l$ for distal link, $u l$ for upper leg, and $l l$ for lower leg.

\subsubsection{Motion Profiles}

Figure 15 shows three different motion profiles performed in simulation: knee bending (A), circling (B), and twisting (C). Videos of the robot performing the motions in simulation 
in real-time are accessible via the Supplementary Material. Profile A moves the torso up and down along the world origin $z$-axis by $z_{O}$, profile $B$ translates the torso in circular motion with radius $r_{O}$ around a fixed centre only in horizontal world $x$-y-axis motion, and profile $C$ rotates the torso around its centre with the angle $\phi_{O}$ without translating the torso. Table 7 displays parameters, characterizing the motion profiles.

The speed respectively frequency $\omega$ that is selected for the motion profiles is limited by several factors and is connected to the fundamental goal of the virtual experiment setup, which is the comparison of the internal loads for both joint topologies. Thus, the specific frequency selected for each profile yields an acceptably close following of the reference trajectory. At this frequency, the joint space PID-control scheme is able to perform the motion profile in a stable fashion. One should note that a different, more robust control law may yield higher speed and accuracy without necessarily more powerful actuators, but for this experiment, the simple implementation works sufficiently. A further aspect at the selection of the frequency $\omega$ is the behaviour of the foot-to-ground contacts. A higher speed may yield slipping of the robot feet, which then would result in possibly different robot poses and motion trajectories for both topologies, invalidating the intended joint load comparison. Additionally, the controller gains selected are tuned under the assumption of a continuous ground contact and would yield mediocre results at a loss of ground contact. Thus, a reasonable frequency $\omega$ was selected for the motion profiles.

profile A
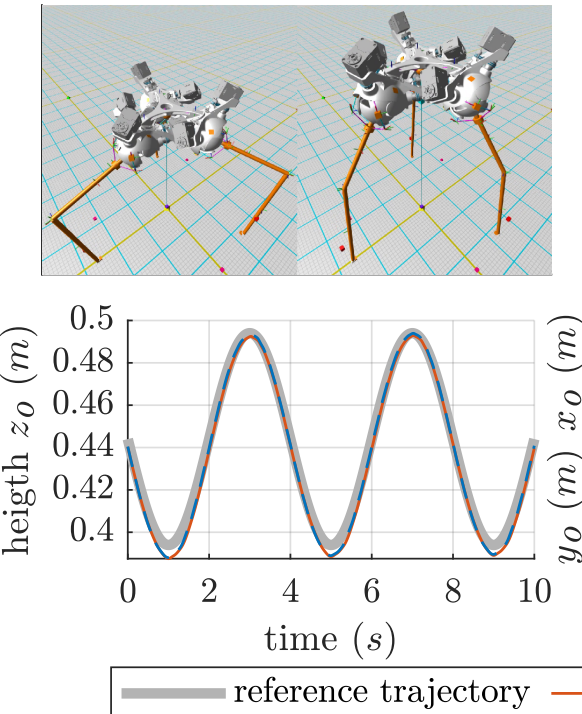

profile B
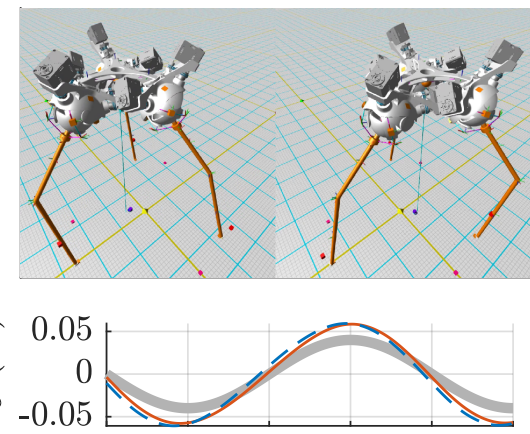

0.05
0
-0.05

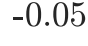

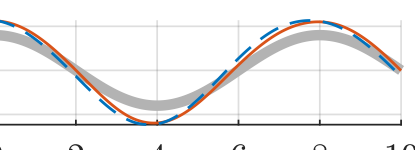

profile $\mathbf{C}$
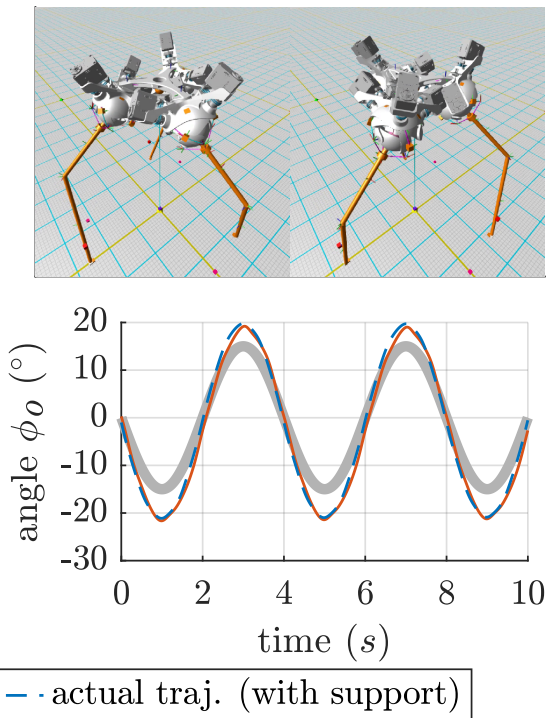

Figure 15. Motion profiles and screenshots of the robot model in simulation, following the reference trajectory. The term with support relates to the configuration $T_{\mathbf{\Delta}}$ with load support joint and no support to the conf. $T_{\triangle}$ without bespoke joint. The screenshots display extrema of the corresponding motion plots.

Table 7. Motion profile parameters, expressing the simulated torso trajectory.

\begin{tabular}{ccccc}
\hline Var. & Unit & Profile A & Profile B & Profile C \\
\hline$h_{t}$ & $\mathrm{~m}$ & 0.444 & 0.482 & 0.485 \\
$r_{f}$ & $\mathrm{~m}$ & 0.246 & 0.246 & 0.246 \\
$\omega$ & $\frac{\mathrm{rad}}{\mathrm{s}}$ & $2 \pi 0.250$ & $2 \pi 0.125$ & $2 \pi 0.250$ \\
\hline$r_{O}$ & $\mathrm{~m}$ & 0 & 0.040 & 0 \\
$x_{O}$ & $\mathrm{~m}$ & 0 & $r_{O} \cos (\omega t)$ & 0 \\
$y_{O}$ & $\mathrm{~m}$ & 0 & $r_{O} \sin (\omega t)$ & 0 \\
$z_{O}$ & $\mathrm{~m}$ & $h_{t}+0.05 \sin (\omega t)$ & $h_{t}$ & $h_{t}$ \\
$\phi_{O}$ & $\mathrm{deg}$ & 0 & 0 & $15 \sin (\omega t)$ \\
\hline
\end{tabular}




\subsubsection{Joint Load Measurement}

By using the Mathworks SimMechanics toolbox [31], the internal joint reaction forces and torques for the motion profiles can be obtained. The load forces and torques are divided in vertical and horizontal components, relative to the joint local reference system. Figure 16 shows the CAD model of the hip unit, which is also shown from the same perspective in Figure 17, but revealing the individual reference frames for the virtual load measurements inside the manipulator mechanism. Horizontal components for forces $f_{R, h}$ and torques $\tau_{R, h}$ are comprised of the red $x$ - and green $y$-axis of the joint reference systems shown in Figure 17, while vertical forces $f_{R, v}$ and torques $\tau_{R, v}$ only contain the component along the blue $z$-axis. In this regard, for this analysism the loads were depicted as

$$
f_{R, h}=\sqrt{f_{R, x}^{2}+f_{R, y}^{2}} \text { and } f_{R, v}=\left|f_{R, z}\right|,
$$

respectively

$$
\tau_{R, h}=\sqrt{\tau_{R, x}^{2}+\tau_{R, y}^{2}} \text { and } \tau_{R, v}=\left|\tau_{R, z}\right|
$$



Figure 16. CAD model of the hip mechanism inside the specially purposed simulation framework for this project.

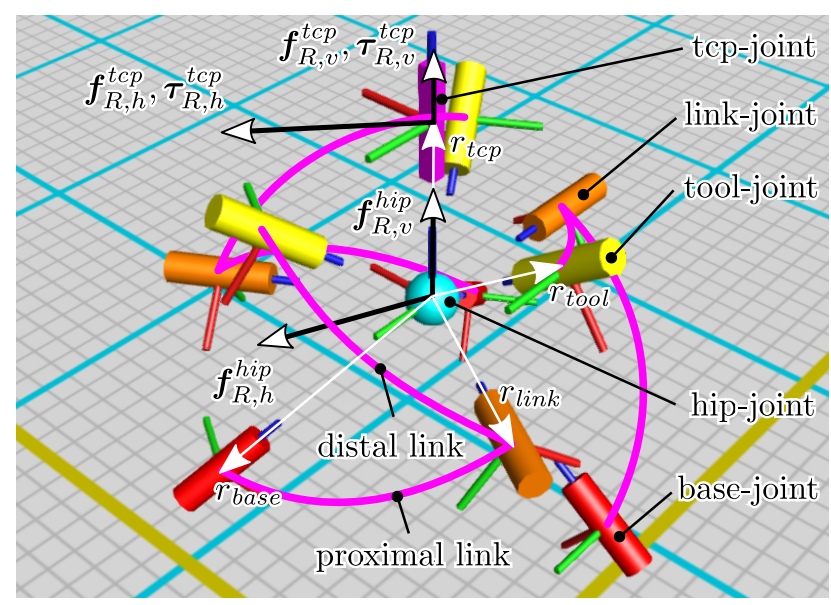

Figure 17. Reference frame view of the hip unit, showing base (red), link (orange), tool (yellow), hip (cyan), and tcp (magenta) joints. Cylinders visualize the joint axis direction. Magenta lines show connections between joints. Red, green, and blue axes represent the orthogonal $x-, y-$, and $z$-axes of the respective reference frames, respectively. 
Hence, the measurement of the hip load takes place in the reference frame located in the centre of the SPM, fixed to the base-platform. Load measurements of the tcp joint were taken from the reference frame fixed to the tool-platform. Since the mechanical model of the manipulator requires multiple spherical shells for collision-free motion in the available workspace, different distances between the linkage joints and the SPM-centre were present. In reality, the mechanical structure of a joint can not be considered as a single point in space. Therefore, a point representative of the transition from one assembly into the next assembly was chosen. For the record, a finite element analysis may reveal the most-demanded location at the joint connection between two assemblies. Hence, Table 8 shows the selected radius of the linkage reference frames respective joint positions for the load measurements relative to the SPM centre. A complete, human-readable file taken from the simulation framework employed in this work, representing the whole robot model, amongst other things, comprising reference frame locations, masses, and inertia matrices, is available via the Supplementary Material.

Table 8. Geometry of SPM joint reference frame placement for load measurements.

\begin{tabular}{ccccc}
\hline Variable & $r_{\text {base }}$ & $r_{\text {link }}$ & $r_{\text {tool }}$ & $r_{\text {tcp }}$ \\
\hline Radius $(\mathrm{m})$ & 0.052 & 0.040 & 0.035 & 0.035 \\
\hline
\end{tabular}

\subsubsection{Controller Setup}

The gains for the PID controller are named $K_{P, b}, K_{I, b}$, and $K_{D, b}$ for the base actuators and $K_{P, k}, K_{I, k}$, and $K_{D, k}$ for the knee actuators. All base actuators share the same setup, despite the fact that an individual setup might deliver better sticking to the reference trajectory. All actuators are limited to an output torque of $\tau_{\max }= \pm 1.2 \mathrm{Nm}$. The gains were selected to yield a well-defined motion profile, tracking the reference trajectory closely, but avoiding a too-aggressive behaviour, which would result in overly high torques and the induction of vibrations into the system. Table 9 lists all controller gains. Furthermore, as a PID control theme was used for a system actually showing pose-dependent nonlinear behaviour, a better fit of the target motion profiles was not achievable with the current controller setup.

Hence, as mentioned in a previous section, the application of the simple joint space PID control law was a limiting factor for the accuracy and performance regarding the quality of the trajectory following that is achievable with this implementation. Consequently, the setup of the robot motion profiles (A, B, and C) was only tuned for the case of a permanent ground contact and the execution of the specific motion profiles. Thus, the later performed walking motion used a different setup for the gains, which enabled a less subtle response to positional errors, resulting in a slightly more overreacting motion in a less-precise fashion, but which is more suitable for the unpredictable walking motion profile.

Table 9. Profile-dependent controller parameters.

\begin{tabular}{ccccccc}
\hline Profile & $K_{P, b}$ & $K_{I, b}$ & $K_{D, b}$ & $K_{P, k}$ & $K_{I, k}$ & $K_{D, k}$ \\
\hline A/B/C & 8.50 & 0.70 & 0.03 & 8.50 & 1.00 & 0.03 \\
Walking & 11.00 & 0.00 & 0.07 & 14.00 & 0.00 & 0.06 \\
\hline
\end{tabular}

\subsection{Walking Analysis}

Since the motivation of this work was to examine the ability of the proposed robot model to perform legged locomotion, an analysis of a walking sequence is important for the further research. Hence, the trajectory controller was created as depicted in Figure 18. Locomotion is achieved by a set of rules, deciding if the leg stays in stance or performs a swing motion. Stance is characterized as the leg being in contact with the ground, carrying the robot weight, whereas the swing moves the foot to the next position without 
having contact to the ground plane. Depending on the desired state, a spatial trajectory is computed and further forwarded to the inverse kinematics solver and the PID controllers.

For simplicity, the leg feet were modelled as point contacts as before, which is important to note, since this has the consequence that a single foot can not resist rotatory motion around its point of contact. As the previous motion profiles rely on permanent contact of all feet with the ground, the emphasis of this circumstance is now important, although the CAD model feet are also very small, hence the difference between real-world behaviour and the simulation will be marginal. With such small contact surfaces in mind, the trajectory controller was configured to always keep two feet on the ground if possible.

\section{Trajectory controller}

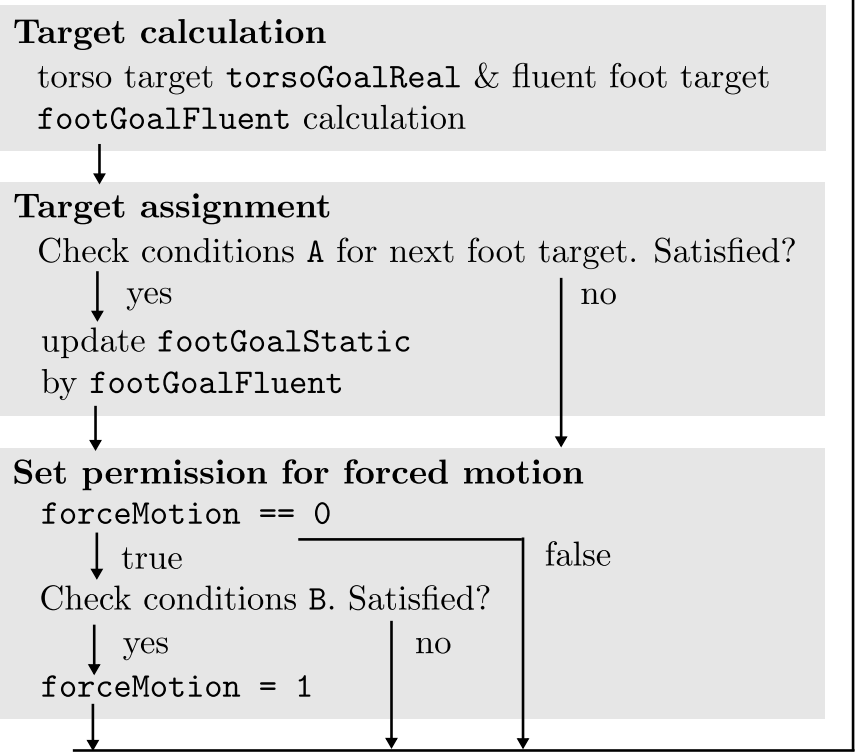

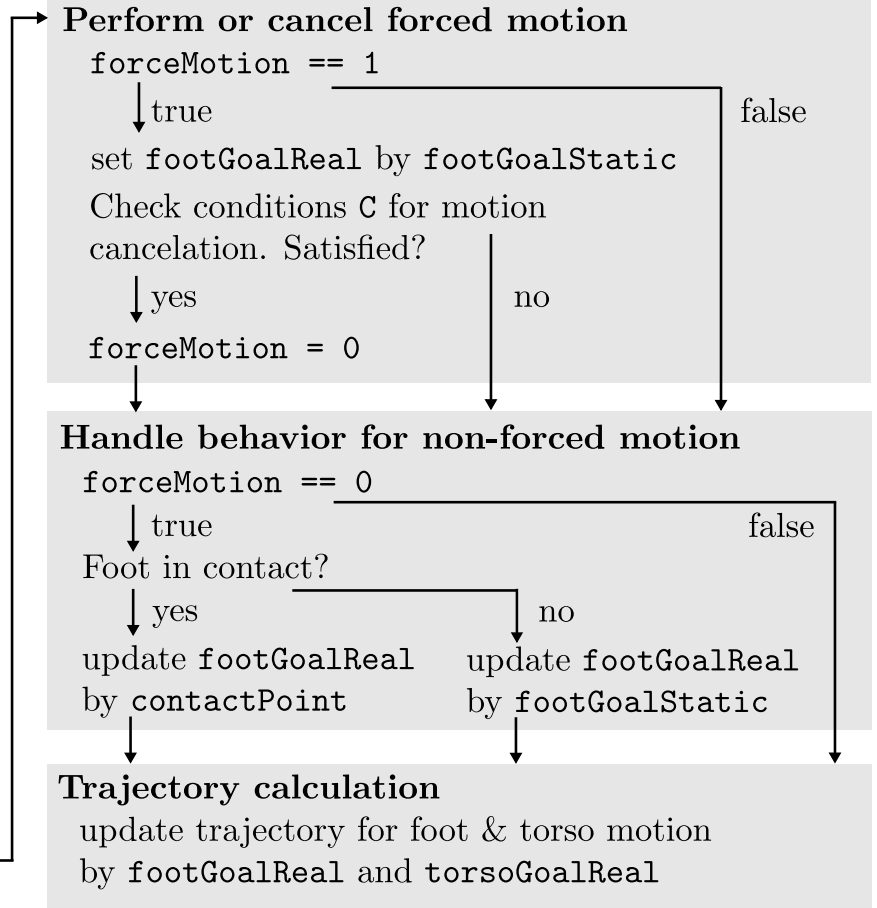

Figure 18. Trajectory controller design for legged locomotion.

As shown in Figure 18, the trajectory controller can be broken down into function groups, starting with the target position and orientation of the robot torso torsoGoalReal and feet footGoalFluent. These values are updated permanently and depend on the walking direction and speed. Since walking is characterized as an alternating sequence of swing and stance states for each leg, based on a set of conditions A, a value footGoalstatic is derived form the footGoalFluent value, working as a fixed target for one foot, being constant for a short period of time. The idea is to always pick a temporarily static value from the continuous target calculation, which is important in regard of providing a fixed target for the trajectory planning, thus yielding more stable motion profiles. Depending on a set of conditions $B$, motion is then carried out for each leg in a forced (forceMotion $=1$ ) or non-forced (forceMotion $=0$ ) way. By dividing into these two motion types, the current state of each leg gets evaluated, and a priority is calculated, yielding the decision regarding which leg has to change its state to the swing mode, as a measure to keep the robot in a stable motion. In the case that no immediate need for the transition to the swing mode exists, the controller either tries to keep the leg in its last initial contact position contactPoint at the collision impact with the ground plane, if in contact, or naturally moves to the next fixed footGoalstatic position. For the situation that a leg is in the swing mode, a set of conditions $\mathrm{C}$ is checked, which is used to cancel any swing motion or forced motion, before letting the robot become unstable. As a preliminary step regarding the current intended and actually reachable target position, the foot target is set to the value footGoalReal inside the function-blocks handling the leg motion, before being used 
in the last step for the actual update of the desired robot trajectory. This final trajectory data are then forwarded into the next controller block for the calculation of the robot inverse kinematics solution.

In the current state of this work, the sets of conditions A, B and C were evaluated by the state of the robot model in simulation. The parameters used for the decision process and the set of rules mainly consist of timing, force, position, speed, and distance information gathered in the previous sensor data interpreter step, which are updated at each simulation iteration and are compared against a manually tuned set of parameters.

\section{Results}

In the following section, the results of the simulations are presented, covering the three motion profiles and the walking sequence. Since all motions of Figure 15 were preformed periodically and were identical for each leg structure, the results depicted in the diagrams only portray one leg without restriction of generality. The resulting plots display the same time frame as depicted in Figure 15 and therefore always cover at least one full period of the motion profile.

\subsection{Load Analysis Results}

Figure 19 shows the load forces and torques of both topologies as depicted in Figure 14 for the motion profiles A, B and C of Figure 15 on the hip and the tcp joint. Since the performed motions of both configurations are still relatively similar in most cases, a comparison between the occurring joint reaction forces and torques is valid.

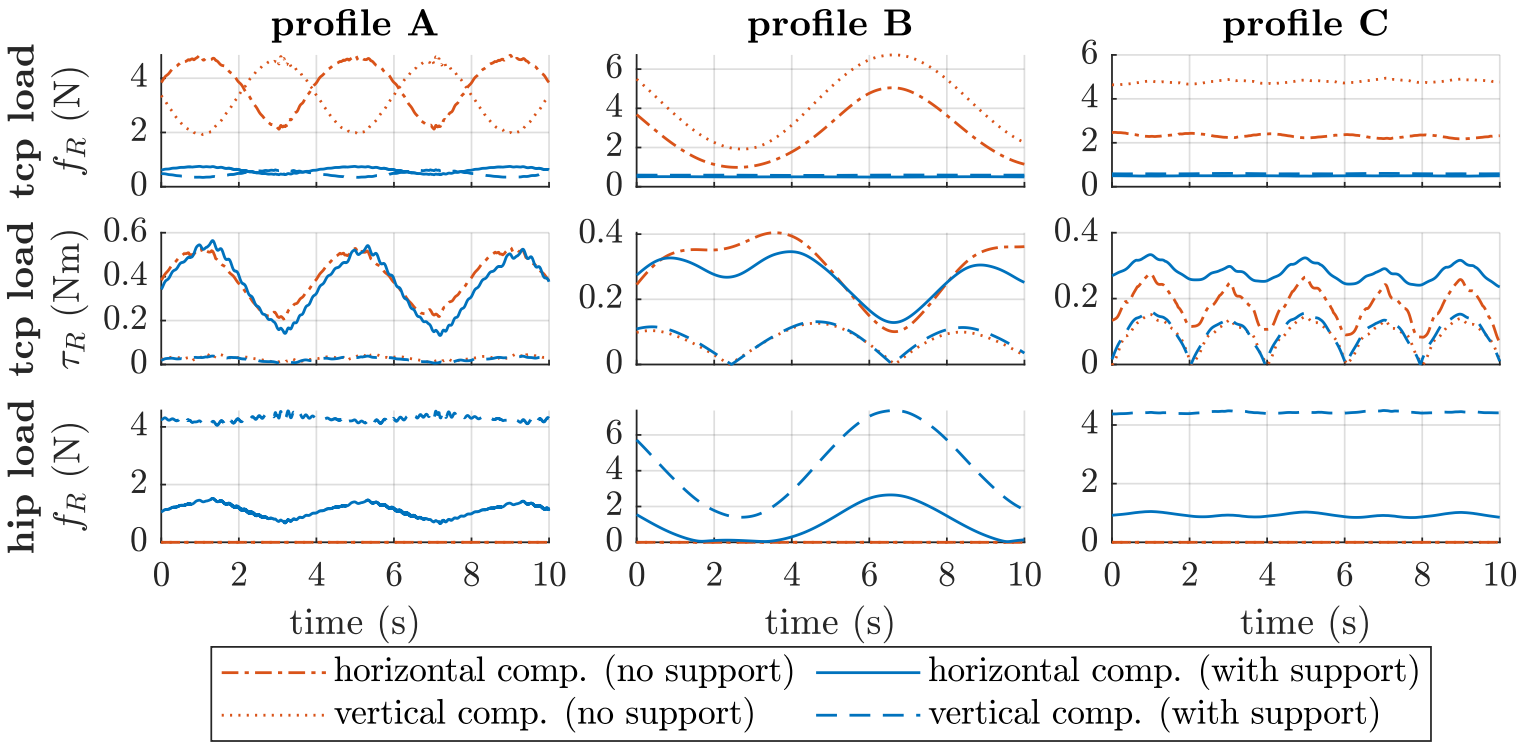

Figure 19. Load forces and torques in horizontal and vertical directions, measured at the hip and the tcp joint for both configurations $T_{\Delta}$ and $T_{\triangle}$.

As can be observed in the diagram, the hip joint at $T_{\boldsymbol{\Lambda}}$ takes a substantial amount of load forces $f_{R}$ in the vertical and horizontal directions, which are otherwise exerted in the tcp joint for the non-support configuration $T_{\triangle}$. Since forces are transmitted into the tcp joint, the tool-platform has to be carried to the full amount by the outer linkage structure in the $T_{\triangle}$ configuration, and the employment of the load support joint therefore highly reduces the overall stress of the manipulator structure. As a result, in the $T_{\boldsymbol{\Delta}}$ case, only a remaining comparatively small force component is applied to the tcp joint, which is related to the connected linkage mass and inertia.

Considering the load torques, a similar picture is apparent for the supportive configuration $T_{\Delta}$ and the non-supporting configuration $T_{\Delta}$, which can be explained by realizing that the demanded torque required to be transmitted over the hip joint is still the same. 
Hence, differences are related only to the topological layout, concerning the linkage structure, that is-regarding the R respectively C SPM base joints-carried to a lesser degree by the base-platform in the $T_{\boldsymbol{\Delta}}$ configuration in contrast to the $T_{\triangle}$ configuration. In this regard, profile $C$ reveals that the twisting motion introduces higher torques into the tcp joint in the $T_{\Delta}$ as in the $T_{\Delta}$ configuration due to the swing motion of the linkage chains itself. These are not supported in the axis direction in $T_{\mathbf{\Delta}}$ by the base-platform anymore due to the cylindrical base joints, thus yielding higher torque loads specifically in these motion profiles. Considering that the torques are still small, this difference is not regarded as concerning.

Regarding a static standing pose of the robot, Table 10 shows an comparison of static forces and torques exerted into the tool-platform for both robot configurations, alongside other measurement data. Since the the standing pose assumes all joint velocities to be zero, the actuator $\underline{\mathrm{C}}$ and $\underline{\mathrm{R}}$ joints are replaced by $\mathrm{P}$ (prismatic) and $\mathrm{W}$ (weld/fixed) joints, without altering the robot system, hence keeping the robot dynamics determinate. By this measure, the actual robot pose (joint coordinates) and actuator torques are identical for both simulated cases. Actuator torques simply are the reaction moments measured at the fixed rotatory DOF of the actuator axes.

The result of this reaction force and torque analysis shows that the load forces introduced in the tool-platform of topology $T_{\Delta}$ in comparison to $T_{\triangle}$ can be reduced to a substantial degree, which allows for thinner shapes of the proximal and distal linkage parts. Table 11 depicts the load reduction ratios $r_{f, \text { load }}$ and $r_{\tau, \text { load }}$, calculated by

$$
r_{f, \text { load }}=\frac{\operatorname{median}\left(\left|f_{R, T_{\Delta}}^{t c p}\right|\right)-\operatorname{median}\left(\left|f_{R, T_{\Delta}}^{t c p}\right|\right)}{\text { median }\left(\left|f_{R, T_{\Delta}}^{t c p}\right|\right)},
$$

based on the joint reaction force vector $f_{R}^{t c p}$ and equally with $\tau_{R}^{t c p}$ for the torque case.

Table 10. Load comparison for $T_{\triangle}$ and $T_{\mathbf{\Delta}}$ with fixed actuator axes, depicting static tripodal standing.

\begin{tabular}{|c|c|c|c|}
\hline Var. & $T_{\triangle}$ & $T_{\boldsymbol{\Delta}}$ & Unit \\
\hline$\left|f_{R}^{t c p}\right|$ & 5.025 & 0.769 & $\mathrm{~N}$ \\
\hline$\left|\tau_{R}^{t c p}\right|$ & 0.801 & 0.876 & $\mathrm{Nm}$ \\
\hline$\tau_{u 1}$ & \multicolumn{2}{|c|}{-0.785} & $\mathrm{Nm}$ \\
\hline$\tau_{u 2}$ & \multicolumn{2}{|c|}{0.371} & $\mathrm{Nm}$ \\
\hline$\tau_{u 3}$ & \multicolumn{2}{|c|}{0.402} & $\mathrm{Nm}$ \\
\hline$\tau_{k}$ & \multicolumn{2}{|c|}{0.259} & $\mathrm{Nm}$ \\
\hline$h_{t}$ & \multicolumn{2}{|c|}{0.513} & $\mathrm{~m}$ \\
\hline$\phi_{1}$ & \multicolumn{2}{|c|}{125.7} & $\circ$ \\
\hline$\phi_{2}$ & \multicolumn{2}{|c|}{125.7} & $\circ$ \\
\hline$\phi_{3}$ & \multicolumn{2}{|c|}{125.7} & $\circ$ \\
\hline$\phi_{k}$ & \multicolumn{2}{|c|}{-22.5} & o \\
\hline
\end{tabular}

Table 11. Load reduction ratios over motion profiles, covering a sequence of $30 \mathrm{~s}$.

\begin{tabular}{cccc}
\hline Load & A & B & C \\
\hline$r_{f, \text { load }}$ & 0.84 & 0.84 & 0.86 \\
$r_{\tau, \text { load }}$ & 0.05 & 0.17 & -0.43 \\
\hline
\end{tabular}

\subsection{Actuator Analysis Results}

The individual actuator torques, $\tau_{u j}$ at the axis $\hat{\boldsymbol{u}}_{j}$ and $\tau_{k}$ at the knee axis, applied to the joints for each motion profile by the PID controller, are depicted in Figure 20a, also for both the supported and non-supported model topology. Figure 20b depicts the corresponding velocity profiles $\dot{\theta}_{u j}$ and $\dot{\theta}_{k}$ of the actuator joint axes. As can be clearly seen in the figures, despite the difference in the topological structure of the robot models, the motion trajectory, 
actuator torques, and velocities are nearly identical. Minor differences can be explained due to the manual triggering of the controller at a certain time in the simulation to start performing the motion profiles, which does result in beginning the measurements from a slightly different systemic state of the robot.

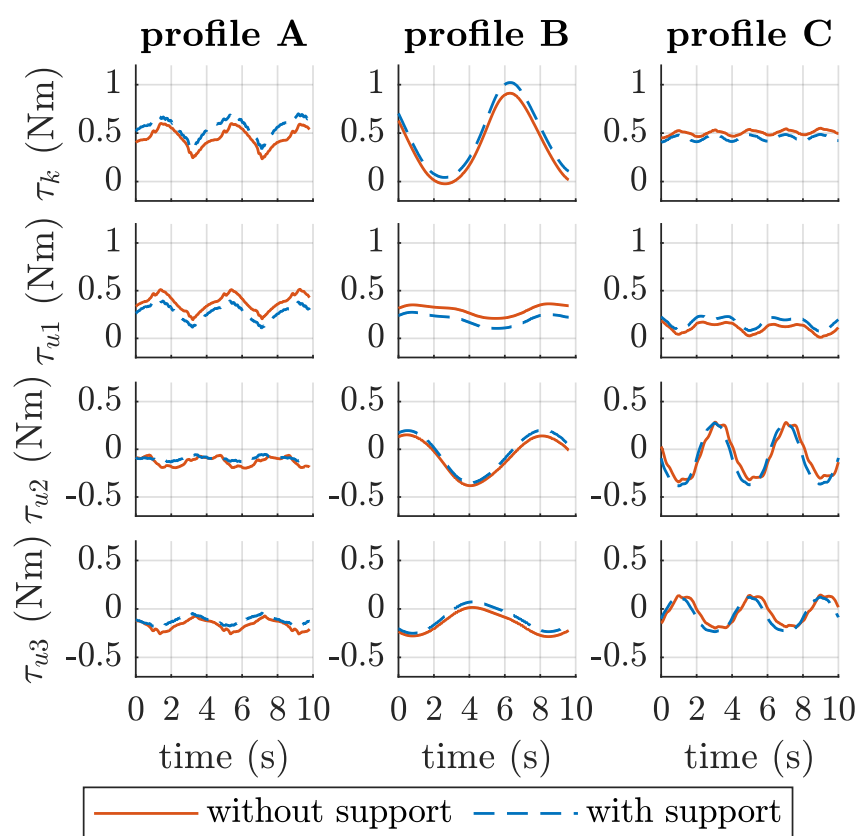

(a)

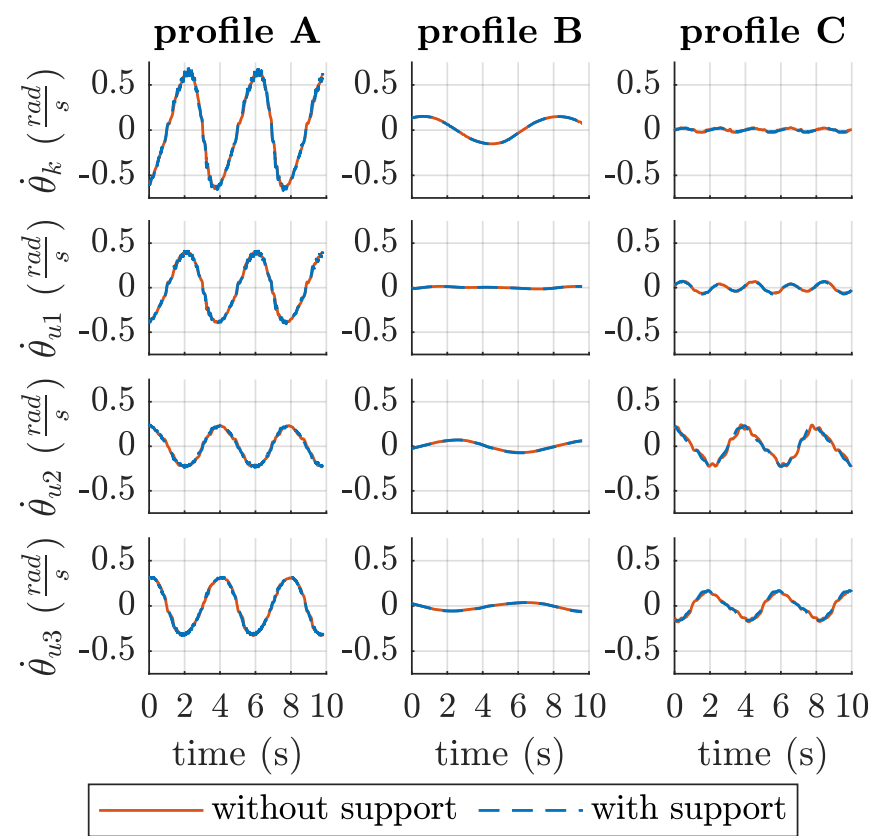

(b)

Figure 20. Required actuator torques (a) and velocities (b) for both robot configurations and each motion profile A, B and C.

The analysis shows that the actuators chosen enable the robot to perform the desired motions, while still providing reasonable performance headroom. Thus, the torque and the velocity profile diagrams show that the actuators were not reaching their respective limits, which are-for the record-a stall torque of $1.9 \mathrm{Nm}$, limited to a continuous torque of $1.2 \mathrm{Nm}$ in the simulation and a maximum no-load velocity of $70 \frac{1}{\mathrm{~min}} \approx 7.33 \frac{\mathrm{rad}}{\mathrm{s}}$ for the employed XC430-W240-T Dynamixel actuators, based on the specifications available at [30]. Since the experiment setup was centred around the stable execution of the motion profiles based on the limiting simple PID control in the robot joint space, the results show that the actuators-respectively their basic performance profiles-were adequately chosen for the specific task.

As the topological structure does not reveal any benefits regarding the measured actuator torque signal, it should be considered that in the case of the 3 RCC configuration, forces are acting in the joint axis direction on the proximal links to the actuator joint, which may increase friction and which are prone to lower the manipulator performance. These forces are not present in the 3CCC configuration and may also allow for a direct motor to link coupling, without any additional passive support bearing, which is used to capture impacts, possibly introduced into the motor shaft in the $3 \underline{R C C}$ or $3 \underline{R R R}$ configuration.

\subsection{Individual Linkage Observation}

Since the general usage of the centre joint is advisable as shown, the actual impact onto the individual linkage chains is considered in the following. Of all motion profiles, the highest reaction forces and torques occurring in the outer linkage structure can be observed in profile B, which therefore are shown in detail in Figure 21. In the figure, the joint loads for both the supported and non-supported configurations are compared, depicting measurements from the same 10-s time frame of the motion profiles. For each 
linkage chain of one of the hip units, the individual base-, link-, and tool-joint loads of the linkages are depicted. Thus, loads are now considered by

$$
f_{R}=\sqrt{f_{R, x}^{2}+f_{R, y}^{2}+f_{R, z}^{2}}, \quad \tau_{R}=\sqrt{\tau_{R, x}^{2}+\tau_{R, y}^{2}+\tau_{R, z}^{2}} .
$$

Load diagrams of all motion profiles are available via the Supplementary Materials and are not included here due to space limitations.
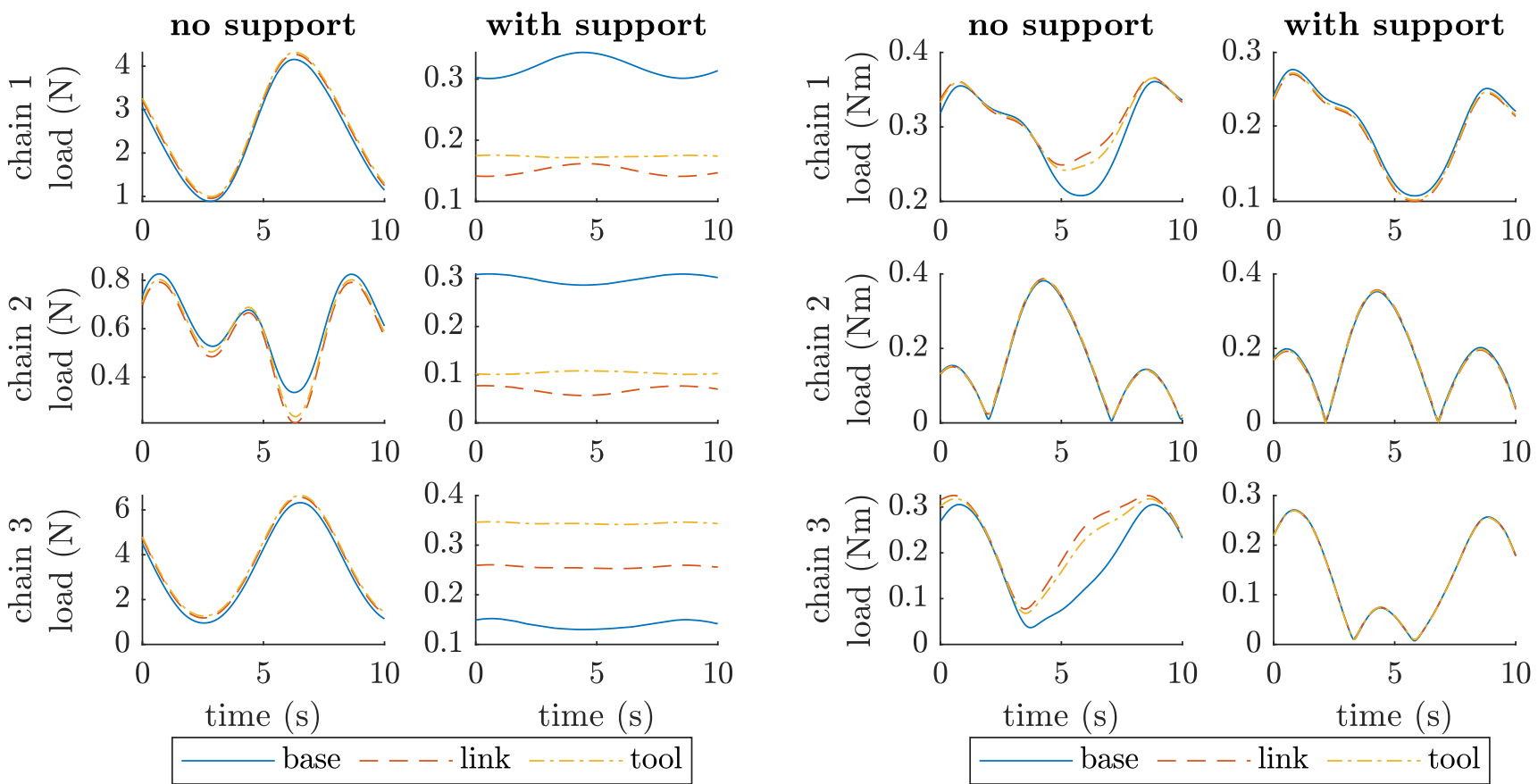

(a)

(b)

Figure 21. Simulated reaction forces (a) and torques (b) of each joint per linkage chain over time for motion profile B.

Regarding the force load diagrams, a quite large reduction of load forces onto the joints was observable between both configurations. Recalling the observation regarding the DOFs of the pure 3CCC linkage in contrast to the $3 \underline{R C C}$ linkage, the $3 \underline{C C C}$ structure is unable to transmit any forces due to its $6 \mathrm{DOF}$ design, requiring a dedicated centre joint. Hence, the actual load distribution inside the linkages is inspected in the following. Since the load support configuration mitigates external forces, differences at the trends for the joints inside the individual linkage chains are present. It should be recalled that the real-world model features a $3 \underline{R} R R$ linkage with slight elasticity inside its links, thus the real model is only an approximation of the simulated one but will still transfer the main part of the external forces over the desired load support joint.

Due to the much smaller scale of the ordinate in these diagrams, the gaps between the individual link joint reaction forces appear more present than in the non-support configuration, despite it not being the case. The reason for the different loads onto the joints can be explained by visualizing the actual topological structure of the system, which, in the case of a simple standing pose of the robot, yields an always-compressed sheerloaded spherical mechanism in the non-support configuration. In contrast, regarding the support case, the spherical linkage is not compressed by the weight of the robot itself, which is carried by the support joint here, and therefore is actually only loaded by its own linkage weight. Thus, regarding the force loads, the individual linkage joints are exerted by the loads, stemming from the weight of its SPM itself, chaining up with each element in the structure, hence explaining different joint loads in the diagrams. In addition, reaction forces are created by the actuator torques, required to exert a certain output torque of the manipulator tool-platform transmitted through the linkages. Realizing that 
torque is transmitted through the spherical structure of the manipulator independently of the existence of the centre joint, which is only able to catch external forces, the similar characteristic of the torque diagrams for both configurations becomes clear. Here, the main difference was found in Chain 3, receiving higher torques due to its substantial amount of external forces transported over the chain in contrast to the non-stressed $T_{\mathbf{\Lambda}}$ version.

\subsection{General Comparison}

A more general comparison of the kinematical requirements and the resulting joint loads including all motion profiles and also the walking sequence are displayed in the boxplots in Figures 22 and 23. Here, the comparison between the motion profiles is considered, while all simulations are based on the supported $T_{\boldsymbol{\Delta}}$ configuration. Since the walking sequence is the only non-symmetrical motion profile considering the employment of the individual legs, data from all hip units were combined for this analysis. For the reduction in the number of measurements, the original signal measured at $100 \mathrm{kHz}$ was filtered with a moving average filter at the span of $10 \mu$ s and downsampled to $100 \mathrm{~Hz}$, before being applied to the boxplots. Each plot is based on an 30-s motion segment.

As it can be observed in the boxplots in Figure 22, the motion profiles take advantage of the individual actuator joints to a varying degree, giving insights to the importance of the individual actuators for certain torso movements. The right side of the plots expresses the orientation of the hip joint in more-representative $x$-y-z-Euler angles. In this depiction, $x$ equals a horizontally oriented joint axis, $y$ the following vertical axis, and $z$ the final twist angles of the tool-platform, hence the hip orientation can be expressed form the base-platform (BP) to the tool-platform (TP) with the rotation matrix ${ }_{B P}^{T P} \boldsymbol{R} \in \mathbb{R}^{3 \times 3}$ around the axes of the coordinate frame by

$$
{ }_{B P}^{T P} \boldsymbol{R}=\boldsymbol{R}_{x}\left(\phi_{x}\right) \cdot \boldsymbol{R}_{y}\left(\phi_{y}\right) \cdot \boldsymbol{R}_{z}\left(\phi_{z}\right) .
$$

In the case ${ }_{B P}^{T P} \boldsymbol{R}=\boldsymbol{I}$, which does stand for the default pose of the SPM-hip unit, the base angles equal $\phi_{i, h o m e}=125.7^{\circ}$. The value is given for reference in conjunction with the values depicted in the diagrams of Figure 22 but is actually arbitrary, since $\phi_{i, h o m e}$ depends on the implementation of the inverse kinematics calculation of the SPM.

\section{distribution of joint angular positions}

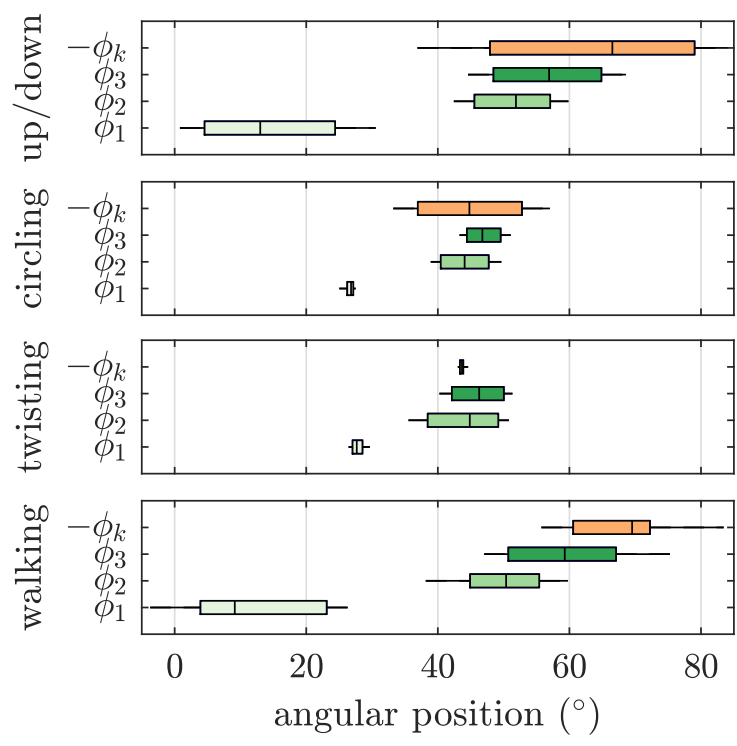

$\phi_{1} \square \phi_{2}$
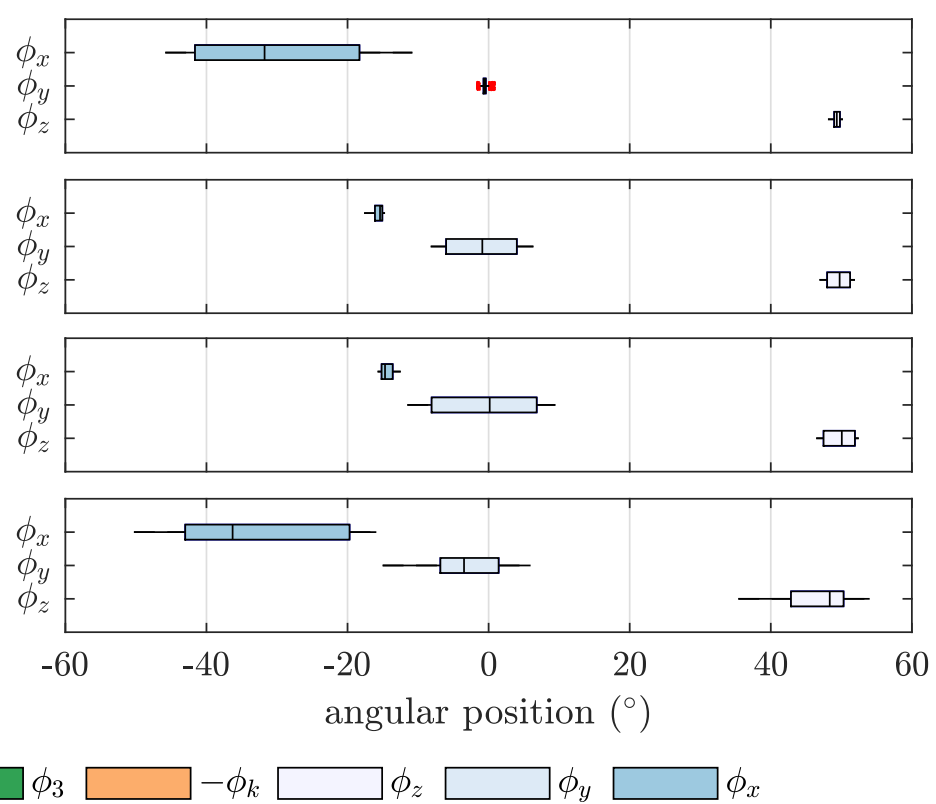

Figure 22. Angular joint distribution for different motion profiles, depicting the actuator joint angles and the resulting hip orientation in Euler angles. For the purpose of compact visualization, data for $\phi_{1}, \phi_{2}$, and $\phi_{3}$ are shifted by $\Delta \phi=-\frac{\pi}{2}$. 
distribution of joint force and torque loads
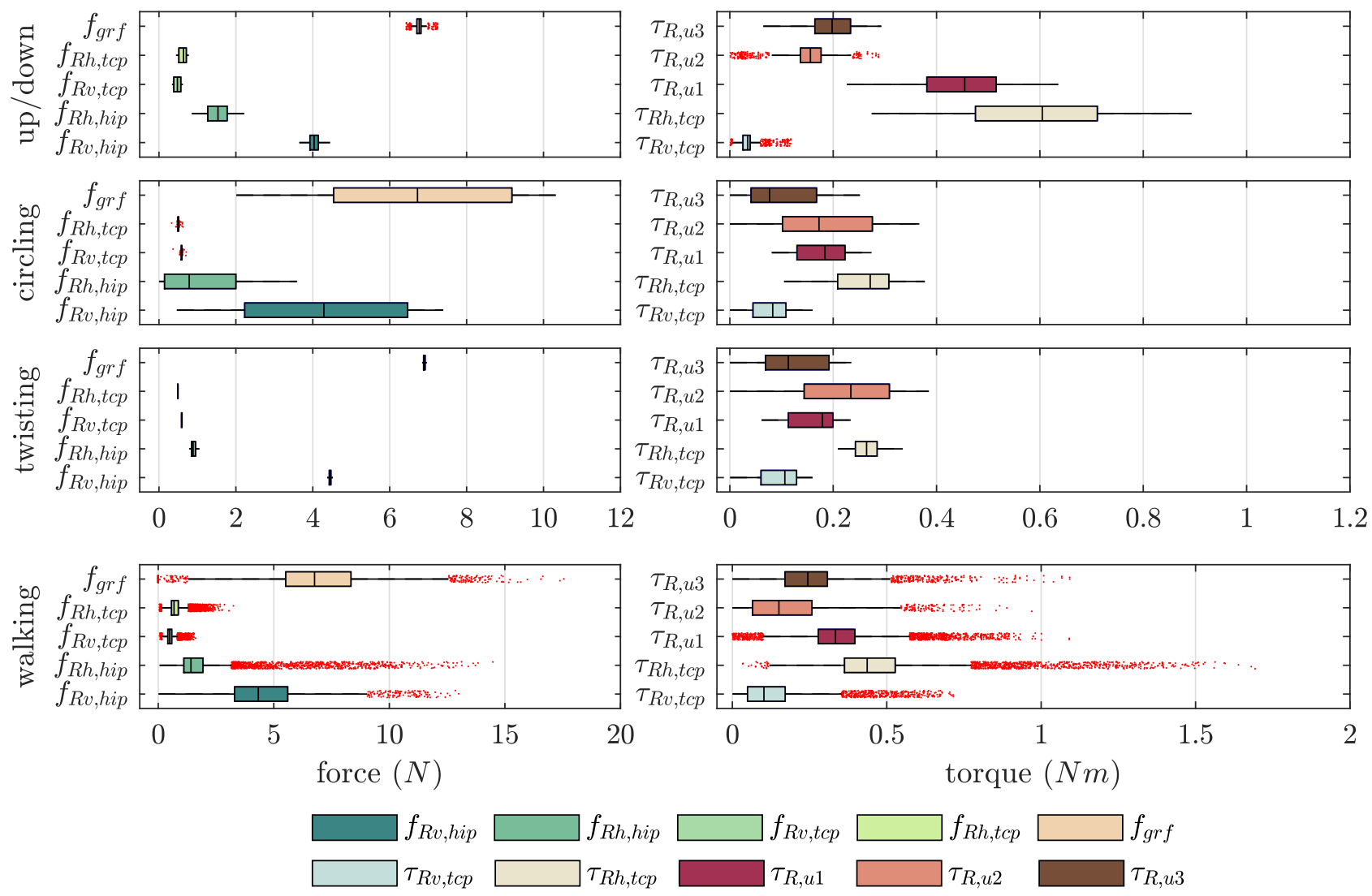

Figure 23. Reaction force and torque of the hip and the tcp joint in the horizontal and vertical component view. $f_{g r f}$ shows ground reaction forces, and $\tau_{R, u i}$ displays the joint reaction torques after regarding actuator torques and joint friction. All values are absolute resp. norms of vector values. A different axis range is used for the walking profile.

It should be mentioned here that the design of a parallel manipulator is motivated to be well-conditioned over its workspace. In this regard, a manipulator should be as close as possible to an isotropic configuration inside its indented workspace ([10], p. 169). The closeness to such an isotropic pose can be expressed by the conditioning index $\eta$ for parallel manipulators, as defined in [14], which shows an isotropic manipulator at $\eta=1$. Here, the conditioning index reveals a slightly non-optimal result for the home configuration $(h)$ of the robot discussed,

$$
\eta\left(\boldsymbol{J}_{h}\right)=\frac{1}{\left\|\boldsymbol{J}_{h}\right\|\left\|\boldsymbol{J}_{h}^{-1}\right\|}=0.978
$$

using the weighted Frobenius norm. The Jacobian $J$ is a matrix depending on the orientation of the vectors $\hat{\boldsymbol{u}}_{j}, \hat{\boldsymbol{v}}_{j}$, and $\hat{\boldsymbol{w}}_{j}$, as also shown in the further sections of this article, which were constructed as described in the following. Hence, in this article, the vector $\hat{\boldsymbol{u}}_{j}$ is defined by

$$
\hat{\boldsymbol{u}}_{j}=\cos \gamma_{u j} \cos \delta_{u j} \hat{\boldsymbol{e}}_{x}+\cos \gamma_{u j} \sin \delta_{u j} \hat{\boldsymbol{e}}_{y}+\sin \gamma_{u j} \hat{\boldsymbol{e}}_{z}
$$

and the vector $\hat{\boldsymbol{w}}_{j}$ accordingly by

$$
\hat{\boldsymbol{w}}_{j}=\cos \gamma_{w j} \cos \delta_{w j} \hat{\boldsymbol{e}}_{x}+\cos \gamma_{w j} \sin \delta_{w j} \hat{\boldsymbol{e}}_{y}-\sin \gamma_{w j} \hat{\boldsymbol{e}}_{z}
$$

with the vertical angles $\gamma_{u j}$ and $\gamma_{w j}$ and with the horizontal orientation angles $\delta_{u j}$ and $\delta_{w j}$ in the hip base reference frame. In the case that an symmetrical arrangement is needed, it can be defined

$$
\delta_{u j}=\frac{j-1}{3} 2 \pi, \quad \text { and } \quad \delta_{w j}=\delta_{u j}+\delta_{i n i t}
$$


with $\delta_{\text {init }}$ setting an initial twist between the base- and the tool-platform. If an angle of $\frac{\pi}{2}$ between neighbouring axes is intended, regarding an optimal configuration as shown in [11], it can be deducted from Figure 24 that it must hold

$$
\gamma_{u j}=\gamma_{w j}=\arccos (\sqrt{2 / 3})
$$

The initial home orientation of the SPM at ${ }_{B P}^{T P} \boldsymbol{R}=\boldsymbol{I}$ is then set by the values shown in Table 12, which also shows an alternative actually ideal configuration of the manipulator. This decision for the non-optimal home orientation of the hip units is based on the mechanical realization, as this would likely produce more internal collisions in other setups with the current compact manipulator layout.

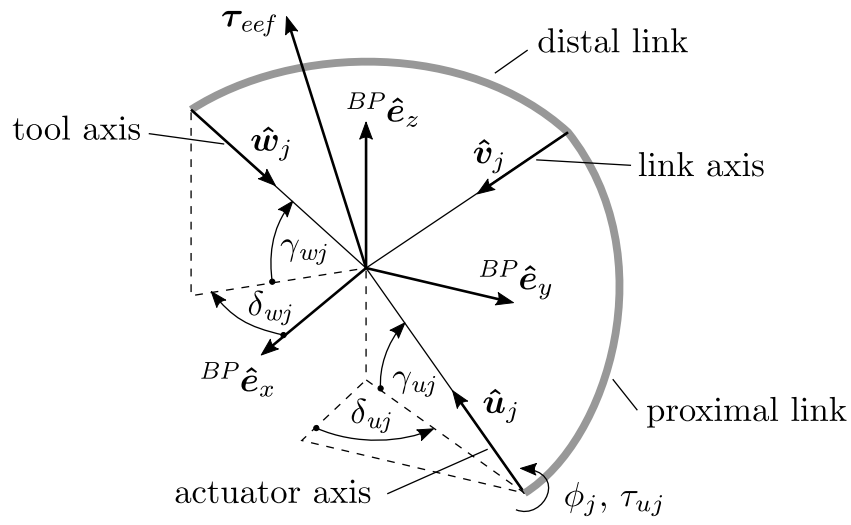

Figure 24. Layout of a single linkage structure.

Table 12. Construction parameters for $\hat{\boldsymbol{u}}_{j}$ and $\hat{\boldsymbol{w}}_{j}$ axes for the SPM home configuration and conditioning index $\eta$.

\begin{tabular}{ccc}
\hline Parameter & Used & Ideal \\
\hline$\gamma_{u j}$ & \multicolumn{3}{c}{$\arccos (\sqrt{2 / 3}) \approx 35.26^{\circ}$} \\
$\gamma_{w j}$ & $\gamma_{u j}$ \\
$\delta_{u j}$ & $0^{\circ}, 120^{\circ}, 240^{\circ}$ & $\delta_{u j}+60^{\circ}$ \\
$\delta_{w j}$ & $\delta_{u j}+45^{\circ}$ & 1.000 \\
$\eta$ & 0.978 & \\
\hline
\end{tabular}

The most evenly distributed usage of the angular range of the individual joint motions was found in the walking sequence, which validates the potential of the SPM to be used for the purpose of walking, since there was not visible any critical non-uniform utilization of the individual joint axes. Regarding the kinematical requirements, both up/down and walking showed similar demands for the joint angles $\phi_{1}, \phi_{2}, \phi_{3}$, and $\phi_{k}$, but this did not reflect into $x-y$-z-Euler representation or the force/torque domain, which reminds that the actual combination of the actuator angles defines the output orientation of the toolplatform. It should be stressed here that all joint angles $\phi$ are actually measured at the plant side and are not to be confused with the reference signal at the controller side. Regarding reaction forces and torques, the walking profile reveals a much wider range of loads, hence showing a certain amount of outliers, although the main accumulation is similar again to the up/down or walking profile. It is noticeable that outliers should not be confused with undesired or corrupted measurements but simply with a non-Gaussian distribution outside of the typical working range, which does occur due to the just-in-time trajectory generation, which, in contrast to the other predetermined motion profiles, does not yield a periodically repeated force/torque characteristic. Moreover, outliers were present in the up/down-motion, which reveals a slight horizontal staggering of the robot while lifting the torso. In general, the force-torque-related boxplots further manifest the importance of the centre joint, which absorbs the leg load, also visible at the measured ground reaction forces 
$f_{g r f}$, which can result in situation-dependent high-peak forces, which must be carried by the hip mechanism.

\subsection{Walking Analysis Results}

Regarding the actual motion profile of the walking sequence of the robot model, Figure 25 displays the swing and stance phases of each leg over a time frame of $10 \mathrm{~s}$. Black bars indicate the foot is performing a swing motion without ground contact, moving to the next target position, otherwise the foot is in contact with the horizontal ground plane.

Since the gait generator does not actively force leg motion in a predefined cycle, the periodicity observable in Figure 25 emerges naturally from the simple priority- respectively rule-based system for the selection of the current stance or swing leg.

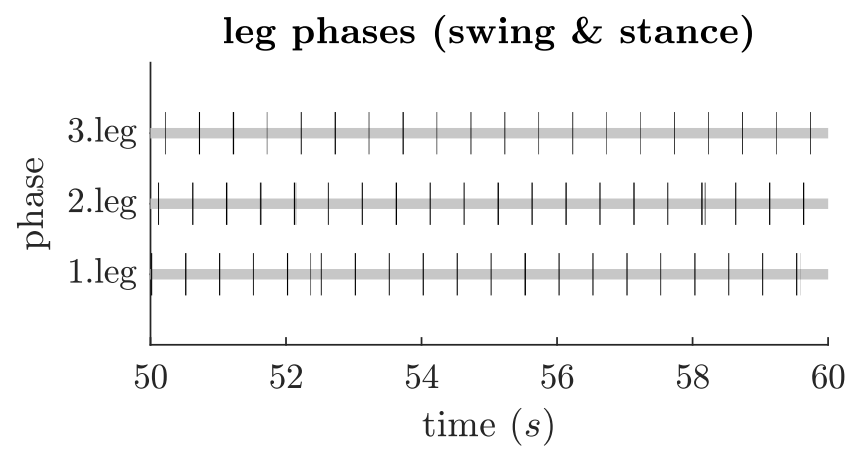

Figure 25. Segment of the walking gait. Black bars indicate swing phases of a particular leg.

As it becomes visible by this depiction, the swing phases are short in time compared to the stance phases, which is a direct result of the mass distribution of the robot model. Since the legs comprise very low masses and inertias, in contrast to the robot torso, the robot legs can be lifted and placed at a new location fast enough before letting the robot become unstable. Fast motion is necessary for this kind of walking, since the three-legged robot is not in a statically stable pose while moving one leg to its next location.

Hence, the contrasting mass and inertia distribution between torso and legs is exploited as the central component of the gait generation, enabling a simple form of locomotion for the conceptual robot model. As can be observed by the ratios $r_{s}$ of leg stance time over the walking sequence duration, as shown in Table 13, the current locomotion keeps the robot mostly in a stable tripodal pose, hence maintaining an statically stable pose, as the projection of the centre of gravity lies within the support polygon, spanned by the three legs in the stance mode.

Table 13. Amount of stance status per leg over the motion sequence.

\begin{tabular}{cccc}
\hline Leg & $\mathbf{1}$ & $\mathbf{2}$ & $\mathbf{3}$ \\
\hline$r_{s}(\%)$ & 96.7 & 96.5 & 96.7 \\
\hline
\end{tabular}

\subsection{Linkage Load Calculation}

Since the designer of the SPM might be interested in the actual load distribution inside the SPM linkages, a more in-depth analysis of the reaction forces and torques due to the exerted actuator torques is required.

\subsubsection{Mathematical Derivation}

As for any manipulator, forces and torques acting on the end-effector can be propagated by means of the kinetostatic analysis, applied from the end-effector back to the base by employing a $6 \times 6$ force-moment-transformation matrix ([26], p. 157-159). Since the load support joint is considered highly stiff in comparison to the slightly elastic linkages parts, along with the mathematical assumption of an outer CCC-structure, it can be 
assumed that any external force is fully captured by the central joint. Hence, the load propagation can be reduced to a $3 \times 3$ matrix calculation, as any external forces and torques injected into the tool-platform result in a pure rotatory motion, only transmitting torque. Due to the spherical structure of the manipulator, the matrix-based moment propagation can furthermore be reduced to a vector calculation, as depicted in Figure 24.

In general, the moment $\tau_{\text {eef }}$ at the tool-platform respectively end-effector (EEF) is supposed to be known, as this is the output moment to be delivered by the manipulator. Considering only EEF torques about the manipulator centre, the only naturally possible direction $\boldsymbol{n}_{j}$ for torques $\tau_{n j}$ to be transmitted by the distal link is normal to the $\hat{\boldsymbol{v}}_{j}-\hat{\boldsymbol{w}}_{j-}$ plane, therefore

$$
\boldsymbol{n}_{j}=\hat{\boldsymbol{w}}_{j} \times \hat{\boldsymbol{v}}_{j}
$$

Hence, the torque vector $\tau_{e e f, j}$ of each linkage acting on the tool platform is related to the end-effector torque vector $\tau_{\text {eef }}$ by

$$
\tau_{e e f}=\sum_{j=1}^{3} \tau_{e e f, j}=\underbrace{\left[\begin{array}{lll}
\frac{n_{1}}{\left|n_{1}\right|} & \frac{n_{2}}{\left|n_{2}\right|} & \frac{n_{3}}{\left|n_{3}\right|}
\end{array}\right]}_{X^{*}} \tau_{d l}
$$

which is a determinate system that delivers the solution for $\boldsymbol{\tau}_{d l}=\left[\begin{array}{lll}\tau_{n 1} & \tau_{n 2} & \tau_{n 3}\end{array}\right]^{T}$, containing the absolute values of the single distal link moments.

Regarding the actuator torques $\tau_{p l}=\left[\begin{array}{lll}\tau_{u 1} & \tau_{u 2} & \tau_{u 3}\end{array}\right]^{T}$ at the proximal links, the projection of the distal link moment onto the actuator axis $\hat{u}_{j}$ can be utilized by

$$
\boldsymbol{\tau}_{p l}=\underbrace{\operatorname{diag}\left(\frac{\boldsymbol{n}_{1}}{\left|\boldsymbol{n}_{1}\right|} \cdot \hat{\boldsymbol{u}}_{1}, \frac{\boldsymbol{n}_{2}}{\left|\boldsymbol{n}_{2}\right|} \cdot \hat{\boldsymbol{u}}_{2}, \frac{\boldsymbol{n}_{3}}{\left|\boldsymbol{n}_{3}\right|} \cdot \hat{\boldsymbol{u}}_{3}\right)}_{\boldsymbol{Y}^{*}} \tau_{d l} .
$$

By rearranging and inserting Equation (18) in Equation (19), this yields

$$
\tau_{p l}=\mathbf{Y}^{*}\left(X^{*}\right)^{-1} \tau_{e e f}
$$

which yields the manipulator Jacobian matrix with

$$
J=\left(\boldsymbol{Y}^{*}\left(\boldsymbol{X}^{*}\right)^{-1}\right)^{T} .
$$

As it can be observed in Equations (18) and (19), it is possible to decompose the matrices $X^{*}$ and $Y^{*}$ into $X^{*}=X D$ and $Y^{*}=Y D$ with the diagonal matrix

$$
\boldsymbol{D}=\operatorname{diag}\left(\left|\boldsymbol{n}_{1}\right|,\left|\boldsymbol{n}_{2}\right|,\left|\boldsymbol{n}_{3}\right|\right) \text {. }
$$

With Equation (21) it follows

$$
\boldsymbol{J}=(\boldsymbol{Y} \underbrace{\boldsymbol{D} \boldsymbol{D}^{-1}}_{\boldsymbol{I}} \boldsymbol{X}^{-1})^{T}=\left(\boldsymbol{Y} \boldsymbol{I} \boldsymbol{X}^{-1}\right)^{T},
$$

with the product of both normal and inverted diagonal matrices $\boldsymbol{D}$ canceling out with the identity matrix $I$. Finally, with the matrices

$$
\begin{aligned}
X & =\left[\begin{array}{lll}
n_{1} & n_{2} & n_{3}
\end{array}\right], \\
Y & =\operatorname{diag}\left(\boldsymbol{n}_{1} \cdot \hat{\boldsymbol{u}}_{1}, \boldsymbol{n}_{2} \cdot \hat{\boldsymbol{u}}_{2}, \boldsymbol{n}_{3} \cdot \hat{\boldsymbol{u}}_{3}\right),
\end{aligned}
$$

it reads for the velocity domain

$$
\boldsymbol{J}=\boldsymbol{X}^{-T} \boldsymbol{Y} \quad \text { and } \quad \boldsymbol{J}^{-1}=\boldsymbol{Y}^{-1} \boldsymbol{X}^{T}
$$


and for the force domain

$$
\boldsymbol{J}^{T}=\boldsymbol{Y} \boldsymbol{X}^{-1} \quad \text { and } \quad \boldsymbol{J}^{-T}=\boldsymbol{X} \boldsymbol{Y}^{-1}
$$

with $*^{-T}$ denoting both matrix inversion and transposition and regarding that for the diagonal matrix $Y$ it must hold $Y=Y^{T}$.

Surprisingly, the author of this study could not find any published similar derivation of the spherical parallel manipulator Jacobian matrix, starting from the force domain, as shown above.

Considering the geometric approach used in [11] to derive the Jacobian $J$ with

$$
J=-A^{-1} B \quad \text { and } \quad J^{-1}=-B^{-1} A
$$

based on the observation that

$$
A \omega+B \dot{\theta}=0
$$

has to be true with the tool-platform angular velocity vector $\omega$ and the actuator axes angular velocity $\dot{\boldsymbol{\theta}}$, it follows that

$$
\boldsymbol{X}^{-T} \boldsymbol{Y}=-\boldsymbol{A}^{-1} \boldsymbol{B}
$$

must hold. By observation and application of the triple product, we can immediately see that

$$
\boldsymbol{Y}=\operatorname{diag}\left(\left(\hat{\boldsymbol{v}}_{1} \times \hat{\boldsymbol{u}}_{1}\right) \cdot \hat{\boldsymbol{w}}_{1},\left(\hat{\boldsymbol{v}}_{2} \times \hat{\boldsymbol{u}}_{2}\right) \cdot \hat{\boldsymbol{w}}_{2},\left(\hat{\boldsymbol{v}}_{3} \times \hat{\boldsymbol{u}}_{3}\right) \cdot \hat{\boldsymbol{w}}_{3}\right),
$$

which is equal to the matrix $\boldsymbol{B}$ found in [11]. Next we observe that

$$
-\boldsymbol{X}^{T}=\left[\begin{array}{c}
\left(\hat{\boldsymbol{v}}_{1} \times \hat{\boldsymbol{w}}_{1}\right)^{T} \\
\left(\hat{\boldsymbol{v}}_{2} \times \hat{\boldsymbol{w}}_{2}\right)^{T} \\
\left(\hat{\boldsymbol{v}}_{3} \times \hat{\boldsymbol{w}}_{3}\right)^{T}
\end{array}\right],
$$

which is identical to $A$ in [11]. To avoid any confusion, please note that the naming convention for the rotatory axes $\hat{\boldsymbol{u}}_{j}, \hat{\boldsymbol{v}}_{j}, \hat{\boldsymbol{w}}_{j}$ in this paper differs from the one found in [11]. Hence, it is shown that it holds

$$
\boldsymbol{Y}=\boldsymbol{B} \quad \text { and } \quad-\boldsymbol{X}^{T}=A .
$$

\subsubsection{Special Case}

Considering the special case with $\alpha_{v w, j}=\pi / 2$, it holds

$$
\hat{\boldsymbol{w}}_{j} \perp \hat{\boldsymbol{v}}_{j} \quad \text { and } \quad\left|\boldsymbol{n}_{j}\right|=1 .
$$

In this situation, due to $\boldsymbol{D}=\boldsymbol{I}$, regarding Equation (22), it becomes

$$
X^{*}=X \quad \text { and } \quad Y^{*}=Y .
$$

Therefore the linkage moment calculation reduces to

$$
\tau_{e e f}=X \tau_{d l} \quad \text { and } \quad \tau_{p l}=\boldsymbol{Y} \tau_{d l} .
$$

\subsubsection{Visual Depiction of Isotropy}

If we consider the manipulator in an isotropic home position, based on the condition that beside requiring orthogonal neighboring vectors $\hat{\boldsymbol{u}}_{j}$ and $\hat{w}_{j}$,

$$
\hat{\boldsymbol{v}}_{j} \perp \hat{\boldsymbol{v}}_{j} \quad \text { and } \quad \hat{\boldsymbol{v}}_{j} \perp \hat{\boldsymbol{u}}_{j},
$$

the manipulator orientation is required to fulfil the condition $n_{j} \cdot \hat{\boldsymbol{u}}_{j}=1$. As shown in [13], there exist several other possible isotropic configurations of the spherical parallel 
manipulator, but, for the current analysis, there was only considered the configuration that has been employed in this study. In this mechanical configuration, each actuator and tool axis is orthogonal to its neighbour axis, and both the proximal and distal links cover an $\alpha_{u v, j}=\alpha_{v w, j}=\frac{\pi}{2}$ arc. Furthermore, the manipulator is set to a pose, where additionally the proximal and distal link planes are oriented vertical to each other. It becomes obvious that, in this case, $\boldsymbol{Y}=\boldsymbol{I}$, and since it holds $\boldsymbol{n}_{j} \| \hat{\boldsymbol{u}}_{j}$ with the vectors $\hat{\boldsymbol{u}}_{j}$ being orthogonal to each other, it must further be $X$ an orthogonal matrix composed of the orthogonal column vectors $n_{j}$. The orthogonality of this matrix also becomes visible at employing $X X^{T}=I$, yielding the identity matrix. Exploiting the properties of orthogonal matrices, it reads for the Jacobian $J_{i s o}$ in the manipulator isotropic pose,

$$
\boldsymbol{J}_{\text {iso }}=\boldsymbol{X}^{-T} \boldsymbol{Y}=\boldsymbol{X} \text {. }
$$

\subsubsection{Visual Depiction of Singularity}

Similarly, if we consider the manipulator being set to a singularity pose, it will hold $\operatorname{det}(\boldsymbol{J})=0$, which obviously is to be avoided in the general case, as this yields additional DOFs for the linkages, without affecting the end-effector pose. In this case, and assuming an ideally shaped manipulator as before, the proximal and distal links are either fully collapsed or extended, which geometrically resolves into $\boldsymbol{n}_{j} \cdot \hat{\boldsymbol{u}}_{j}=0$. In consequence, at least one diagonal element of $\boldsymbol{Y}$ becomes zero, rendering the Jacobian $\boldsymbol{J}$ non-invertible.

\subsubsection{Numerical Considerations}

In any case, the Jacobian $J$ is a configuration-dependent matrix, which has to be recalculated in a moving manipulator for any instance, in which the relation between input and output values for the force or velocity domain is requested. In this sense, it should be considered to not explicitly calculate the Jacobian matrix $J$ by either Equation (26) or (28), as this may be slow and numerically inaccurate in actual controller implementations. Instead, by employing Equations (18) and (19) after each other, the system of equations may be solved by Gauß elimination, yielding the required vector, without explicitly calculating the manipulator Jacobian matrix.

If we consider the case, in that one wants to calculate the actuator joint velocity $\dot{\phi}$ based on the EEF-velocity $\omega$ by

$$
\dot{\phi}=J^{-1} \omega
$$

we can observe that due to the vector construction of $\boldsymbol{X}$ and $\boldsymbol{Y}$, a compact notation is possible,

$$
\dot{\boldsymbol{\phi}}=\left[\begin{array}{lll}
\frac{n_{1}}{n_{1} \cdot \hat{u}_{1}} & \frac{n_{2}}{n_{2} \cdot \hat{u}_{2}} & \frac{n_{3}}{n_{3} \cdot \hat{u}_{3}}
\end{array}\right]^{T} \boldsymbol{\omega} .
$$

This notation only requires one instead of two vector cross products per column in comparison to the calculation based on $J^{-1}$ from Equation (28). Due to the repeated use of vector $\boldsymbol{n}_{j}$, the specific implementation in Equation (40) might require less computational effort, but the actual computational performance will also be highly language- and implementation-dependent. In this regard, Table 14 lists the time needed for the numerical calculation of the actuator velocity based on a given randomized EEF-velocity for $n=10^{8}$ samples, depicting four different implementations of Equation (39).

Method $A$ employs the Matlab inv() operator, which calculates the matrix inverse by LU-decomposition, and operates on the matrices $\boldsymbol{A}$ and $\boldsymbol{B}$ from Equation (28); $B$ also uses $\boldsymbol{A}$ and $\boldsymbol{B}$ from Equation (28) but actually solves the linear system of equation by the Matlab $\backslash$ operator. $C$ rearranges Equation (28) into the shortest possible single matrix expression, while $D$ uses Equation (40). The Matlab code is available via the Supplementary Materials. As it becomes obvious, the specifically tailored expressions of $C$ and $D$ show the fastest calculation, which suggests that in an actual controller implementation, one must be careful regarding the Jacobian construction. 
Table 14. Average execution time on Intel i5-8365U CPU, based on $n=10^{8}$ samples, measured for calculating the solution to Equation (39).

\begin{tabular}{lc}
\hline Method & Time ( $\boldsymbol{\mu s})$ \\
\hline (A) Equation (28) w. Matlab inv() operator & 16.772 \\
$(B)$ Equation (28) w. Matlab $\backslash$ operator & 12.189 \\
(C) Equation (28) in max. compact notation & 10.118 \\
$(D)$ Equation (40) with reused vector $\boldsymbol{n}_{j}$ & 9.937 \\
\hline
\end{tabular}

\subsubsection{Internal Linkage Stress}

Stress transmitted inside the linkage material due to the applied torques is revealed by the equivalent force or pressure as a function of the linkage radius. Hence, regarding any vector position along the distal link, which may be constructed with the angle-axis rotation matrix $\boldsymbol{R}_{n}$ by

$$
\boldsymbol{r}_{d l, j}=\boldsymbol{R}_{n}\left(\beta_{v w, j}, \frac{\boldsymbol{n}_{j}}{\left|\boldsymbol{n}_{j}\right|}\right)\left(-\hat{\boldsymbol{w}}_{j}\right),
$$

with $\beta_{v w, j} \in\left[0, \alpha_{v w, j}\right]$ and building the skew symmetric matrix $\boldsymbol{M}(\boldsymbol{r})$,

$$
\boldsymbol{M}(\boldsymbol{r})=\left[\begin{array}{ccc}
0 & -r_{z} & r_{y} \\
r_{z} & 0 & -r_{x} \\
-r_{y} & r_{x} & 0
\end{array}\right],
$$

the internal link force vector $f_{d l, j}$ is related by

$$
\boldsymbol{\tau}_{e e f, j}=\boldsymbol{M}\left(\boldsymbol{r}_{d l, j}\right) f_{d l, j}
$$

with the EEF torque being known from Equation (18) as

$$
\boldsymbol{\tau}_{e e f, j}=\tau_{n j} \frac{\boldsymbol{n}_{j}}{\left|\boldsymbol{n}_{j}\right|} .
$$

As a consequence of the matrix $M(r)$ not having a full column rank, the system in Equation (43) is underdetermined, which then is solved by the minimum norm least squares solution, which aims to also minimize $\|f\|$. Minimizing the force vector always yields the shortest possible vector, which is directed orthogonal to both lever $\boldsymbol{r}$ and torque $\boldsymbol{\tau}$. Since the direction of the force vector is known to be orthogonal to the radius vector and the torque direction, the solution may also be constructed directly by pure geometric observation.

Considering the proximal link force distribution, it is suitable to observe the linkage stress as a consequence of actuator torque and joint reaction forces. In this regard, the vector $\boldsymbol{m}_{j}$, orthogonal to the $\hat{\boldsymbol{u}}_{j}-\hat{\boldsymbol{v}}_{j}$-plane,

$$
\hat{\boldsymbol{m}}_{j}=\frac{\hat{\boldsymbol{v}}_{j} \times \hat{\boldsymbol{u}}_{j}}{\left|\hat{\boldsymbol{v}}_{j} \times \hat{\boldsymbol{u}}_{j}\right|}
$$

and the vector $\hat{\boldsymbol{g}}_{j}$ with $\hat{\boldsymbol{g}}_{j} \perp \hat{\boldsymbol{u}}_{j}$ and $\hat{\boldsymbol{g}}_{j} \perp \hat{\boldsymbol{m}}_{j}$,

$$
\hat{\boldsymbol{g}}_{j}=\hat{\boldsymbol{u}}_{j} \times \hat{\boldsymbol{m}}_{j}
$$

is constructed, shaping a reference frame fixed to the proximal link centre. Thus, employing the projection of the vector $\tau_{e e f, j}$ onto the frame axes,

$$
\boldsymbol{\tau}_{p r, j}=\left[\begin{array}{lll}
\hat{\boldsymbol{u}}_{j} & \hat{\boldsymbol{m}}_{j} & \hat{\boldsymbol{g}}_{j}
\end{array}\right]^{T} \boldsymbol{\tau}_{e e f, j},
$$


the EEF torque is split into the vectors

$$
\begin{aligned}
\boldsymbol{\tau}_{u, j} & =\boldsymbol{\tau}_{p r, x, j} \hat{\boldsymbol{u}}_{j}, \\
\boldsymbol{\tau}_{m, j} & =\boldsymbol{\tau}_{p r, y, j} \hat{\boldsymbol{m}}_{j}, \\
\boldsymbol{\tau}_{g, j} & =\boldsymbol{\tau}_{p r, z, j} \hat{\boldsymbol{g}}_{j} .
\end{aligned}
$$

With the general construction of a vector function $r_{\perp}$ orthogonal to the corresponding split torque vector direction $\hat{d}$ of unit length,

$$
r_{\perp}(r, \hat{d}):=r-(r \cdot \hat{d}) \hat{d},
$$

it is possible to calculate the equivalent linkage forces for the vector position

$$
\boldsymbol{r}_{p l, j}=\boldsymbol{R}_{n}\left(\beta_{u v, j}, \hat{\boldsymbol{m}}_{j}\right)\left(-\hat{\boldsymbol{v}}_{j}\right),
$$

with $\beta_{u v, j} \in\left[0, \alpha_{u v, j}\right]$ again as the minimum norm least squares solution to

$$
\begin{aligned}
\tau_{u, j} & =\boldsymbol{M}\left(\boldsymbol{r}_{\perp}\left(\boldsymbol{r}_{p l, j}, \hat{\boldsymbol{u}}_{j}\right)\right) \boldsymbol{f}_{u j}, \\
\boldsymbol{\tau}_{m, j} & =\boldsymbol{M}\left(\boldsymbol{r}_{\perp}\left(\boldsymbol{r}_{p l, j}, \hat{\boldsymbol{m}}_{j}\right)\right) \boldsymbol{f}_{m j}, \\
\boldsymbol{\tau}_{g, j} & =\boldsymbol{M}\left(\boldsymbol{r}_{\perp}\left(\boldsymbol{r}_{p l, j}, \hat{\boldsymbol{g}}_{j}\right)\right) \boldsymbol{f}_{g j} .
\end{aligned}
$$

Figure 26 displays the result of the linkage force projection for an exemplary case. In this regard, the distribution of internal forces may guide the designer of the linkages, regarding the required profile of the links.

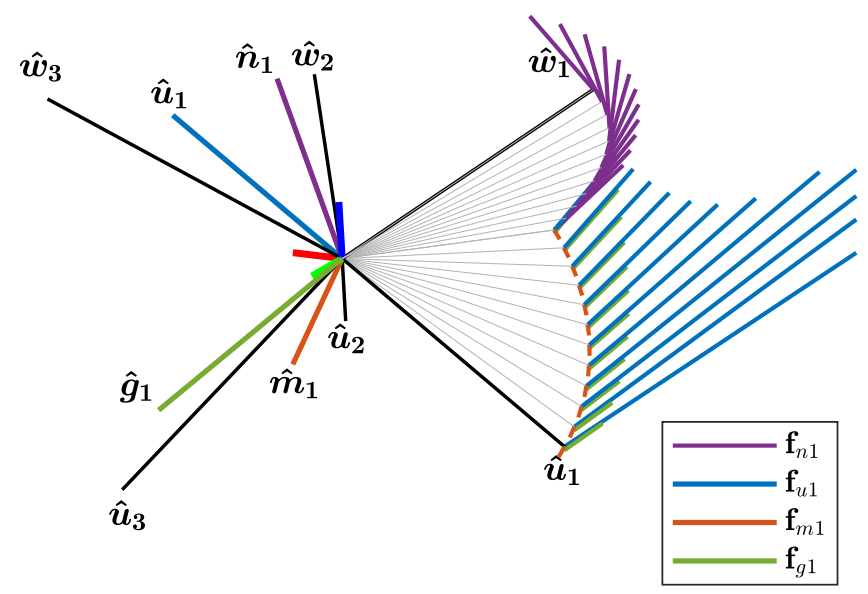

Figure 26. Internal forces along the proximal and distal link for the EEF-torque $\tau_{e e f}=-1 \mathrm{Nm} \hat{\boldsymbol{e}}_{z}$. Link arc angles are set to $\alpha_{u v}=\alpha_{v w}=60^{\circ}$ for visualization purposes.

\section{Discussion}

The results obtained in this study confirm the importance of the load support joint for the spherical parallel manipulator employed as a compact and lightweight hip joint in walking robots. By using the load support joint, stress on the individual linkage structures of the SPM can be effectively reduced, allowing for a small and compact manipulator design. Since the linkage structure is decoupled from external forces at foot-to-ground impacts and the actual weight of the robot itself, the focus of the linkage structure can be shifted towards the transmission of torques by the actuators and external torques introduced in the tcp joint. For this work, this is shown by the build prototype that reveals that proximal and distal links can be designed as thin but spherical shaped parts, which allows for the 
primary transmission of forces tangential to the spherical surface, since there is no need to withstand surface vertically directed forces in the 3CCC concept.

Furthermore, and relying on the existence of the the force absorbing centre joint, the overall stiffness and strength of the manipulator against distortion can be increased, since the centre of rotation is defined by a single existing support joint, in contrast to the combined arrangement of the parallel outer structure in a usual spherical manipulator. Additionally, due to the fully defined centre of rotation, the outer 3CCC structure allows for lower tolerances regarding the manufacturing process, since the actual radius of the linkage joints towards the centre is no longer relevant in contrast to the non-supported 3RCC or overconstrained 3RRR manipulator. Since the manipulator is designed for fast movements, the lower load forces on the linkage joints will naturally show less friction, increasing the obtained manipulator performance.

With this result in mind, the load support joint is of high significance for the design of the robot model but may also limit the motion capabilities of the robot hip, since the support joint requires a solid connection between the hip joint and the SPM-base-platform. This connection may interfere with the local workspace requirements of the shape of the proximal and distal link bodies and has to be considered in the design process. Depending on the desired workspace of the manipulator, this may be a critical drawback, which has to be regarded, concerning the possibility for sudden self-collisions of the mechanism.

Another important result obtained from the experiments is the demonstrated usability of the SPM in the close interlocking arrangement that shows the possibility to achieve suitably dexterity at the performed motion profiles. The results show that neither the kinematics nor the torque profiles reveal requirements that are mechanically impossible or outside of realistically deliverable actuator power. Thus, the SPM is a promising candidate regarding optimal and dense mass distribution in regard to achieving a robot design that delivers high agility with light and fast moving legs.

Regarding the joint space PID-control law employed, several results are notable. Since the control law is not mature enough to properly deal with the robot's non-linear behaviour, two setups were applied to the robot: one for the walking sequence and one for the motion profiles. While both setups are tuned for the specific task and thus perform reasonably well, the control law is quite fragile when it comes to unpredicted situations. Thus, any external disturbances may alter the systemic state to a degree, in which the joint space PID-control-scheme is not able to stabilize the system anymore.

As a final note regarding the discussion, the provided mathematical derivation of the force distribution inside the linkages itself may be useful in the actual design phase of the SPM construction. Depending on the intended motion profile that is desired to be performed by the SPM and the actual external load that is present, a non-symmetrical shaping of the proximal and distal links may be regraded, which can be done under the evaluation of the equations derived in this study.

\section{Conclusions and Future Work}

In this study, mechanical design topologies for the utilization of the spherical parallel manipulator for the implementation in walking robots were investigated. Simulations were performed and discussed, while a general three-legged omnidirectional design concept for a walking robot employing the SPM as a hip joint was developed, targeting a close match of the SLIP model. The applied joint space PID-control law-specifically tailored for the very specific task shown in this study - showed sufficient results, but, as a takeaway, future development iterations of the robot will require a more robust control law implementation that is able to adapt for the complex dynamical properties of the legged robot. Thus, as part of future research, the focus on a more sophisticated control law is prioritized highly for being able to achieve robust locomotion performance and the possibly intelligent exploitation of the robot topology, regarding the optimization of aspects like energy usage, agility, speed, and adaptivity to uneven terrain. Since the conceptual work shows promising 
results, the development of an actual complete robot prototype is now the main focus of future work.

Hence, based on the findings discussed in this study, a final version of the robot was designed that addresses issues revealed. Figure 27 shows the CAD model of the refined robot that is currently in development, which features a complete robot assembly without any placeholder parts as in the conceptual model. The new model and its design specifics will be discussed in a separate article, but a few key details are mentioned in the following. A major addition in comparison to the conceptual model is the addition of series elasticities inside the leg mechanism, enabling the development of an energetically efficient and natural gait, as springs provide the possibility for energy storage while walking, hence opening up the robot to employ passive dynamics in combination with possibly reduced active actuation. As it becomes apparent by observing the topology of this refined model, a series combination of parallel mechanism structures was used, yielding a hybrid serial-parallel robot model.

The model therefore comprises an internal four-bar closed-loop mechanism between the tool-platform and the lower leg, yielding the required additional DOF for the leg elasticity. Moreover, in regard to matching the SLIP model, the knee actuator was moved inside the torso and connected via an articulable chain to the lower leg. In more detail, the actual bending part of the chain that goes through the centre of the hip joint is actually made up of two miniature homokinetic Rzeppa joints, while the part located inside the upper leg is a slider mechanism, comprising a rotatory to linear motion screw, which connects to the knee joint. To accomplish the central axis structure, the load support joint was replaced by a universal joint that shares its distal revolute axis with the integrated parallel mechanism for the realization of leg compliance. A future article will discuss the design and geometric optimization strategy for the refined robot model in detail and will also discuss possible control law implementations as an important aspect for the goal of realizing legged locomotion.

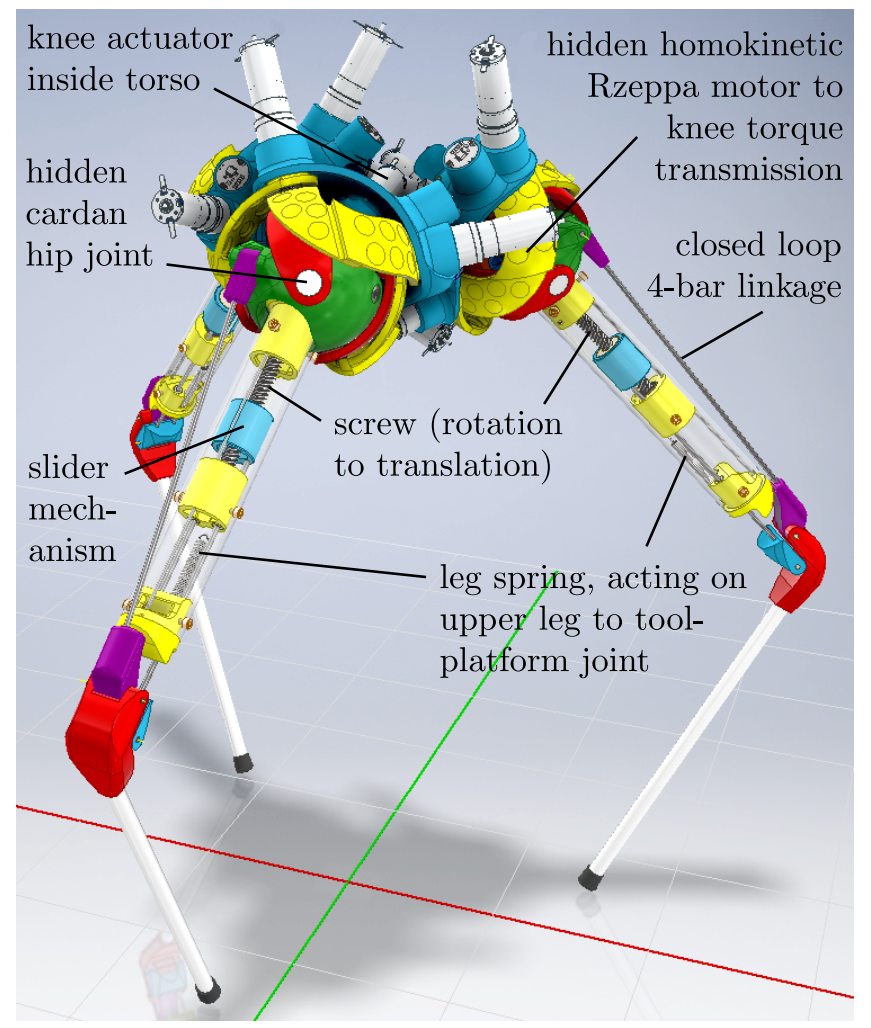

Figure 27. Refined current robot model, including leg elasticities. 
Supplementary Materials: The following Supplementary Materials are available online at https: / / www.mdpi.com/article/10.3390/robotics10040117/s1 respectively [32]. File: Human-readable robot model framework file. Files: Matlab scripts for the Jacobian time measurements. Figures: The complete linkage load diagrams. Videos: Videos of the robot performing profiles A, B, and C and walking.

Author Contributions: Conceptualization, D.F.; methodology, D.F.; software, D.F.; validation, D.F.; formal analysis, D.F.; investigation, D.F.; resources, D.F.; data curation, D.F.; writing-original draft preparation, D.F.; writing-review and editing, D.F.; visualization, D.F.; supervision, D.F. and C.S.; project administration, D.F.; funding acquisition, D.F. All authors have read and agreed to the published version of the manuscript.

Funding: This research received no external funding.

Acknowledgments: The author wants to thank CeramTec [29] for providing the BIOLOX forte [35] joint without charge to support this research.

Conflicts of Interest: The authors declare no conflict of interest.

\section{References}

1. Geyer, H.; Saranli, U. Gait Based on the Spring-Loaded Inverted Pendulum. Humanoid Robot. A Ref. 2017. [CrossRef]

2. Blickhan, R. The spring-mass model for running and hopping. J. Biomech. 1989, 22, 1217-1227. [CrossRef]

3. McMahon, T.; Greene, P. Fast running tracks. Sci. Am. 1978, 239, 148-163. [CrossRef] [PubMed]

4. Garcia, E.; Arevalo, J.C.; Munoz, G.; Gonzalez-de Santos, P. On the Biomimetic Design of Agile-Robot Legs. Sensors 2011, 11, 11305-11334. [CrossRef] [PubMed]

5. Raibert, M.H. Legged Robots that Balance; MIT Press: Cambridge, MA, USA, 1986.

6. Grimes, J.; Hurst, J. The design of ATRIAS 1.0: A unique monopod, hopping robot. In Proceedings of the 15th International Conference on Climbing and Walking Robots and the Support Technologies for Mobile Machines (CLAWAR), Baltimore, MD, USA, 23-26 July 2012; pp. 548-554. [CrossRef]

7. Saranli, U.; Buehler, M.; Koditschek, D. Design, modeling and preliminary control of a compliant hexapod robot. In Proceedings of the Proceedings 2000 ICRA. Millennium Conference, IEEE International Conference on Robotics and Automation, San Francisco, CA, USA, 24-28 April 2000; Volume 3, pp. 2589-2596. [CrossRef]

8. Ren, P.; Hong, D. Triple Stance Phase Displacement Analysis With Redundant and Nonredundant Sensing in a Novel ThreeLegged Mobile Robot Using Parallel Kinematics. J. Mech. Robot. 2009, 1, 041001. [CrossRef]

9. Oki, K.; Ishikawa, M.; Li, Y.; Yasutani, N.; Osuka, K. Tripedal walking robot with fixed coxa driven by radially stretchable legs. In Proceedings of the 2015 IEEE/RSJ International Conference on Intelligent Robots and Systems (IROS), Hamburg, Germany, 28 September-2 October 2015; pp. 5162-5167. [CrossRef]

10. Merlet, J. Parallel Robots; Solid Mechanics and Its Applications; Springer: Berlin/Heidelberg, Germany, 2005.

11. Gosselin, C.; Angeles, J. The Optimum Kinematic Design of a Spherical Three-Degree-of-Freedom Parallel Manipulator. J. Mech. Transm. Autom. Des. 1989, 111, 202-207. [CrossRef]

12. Gosselin, C.; Angeles, J. A Global Performance Index for the Kinematic Optimization of Robotic Manipulators. J. Mech. Des. 1991, 113, 220-226. [CrossRef]

13. Gosselin, C.M.; Lavoie, E. On the Kinematic Design of Spherical Three-Degree-of-Freedom Parallel Manipulators. Int. J. Robot. Res. 1993, 12, 394-402. [CrossRef]

14. Gosselin, C.M.; St. Pierre, E.; Gagne, M. On the development of the Agile Eye. IEEE Robot. Autom. Mag. 1996, 3, 29-37. [CrossRef]

15. Huang, Z.; Yao, Y.L. A new closed-form kinematics of the generalized 3-DOF spherical parallel manipulator. Robotica 1999, 17, 475-485. [CrossRef]

16. Gosselin, C.M.; Hamel, J. The agile eye: A high-performance three-degree-of-freedom camera-orienting device. In Proceedings of the 1994 IEEE International Conference on Robotics and Automation, San Diego, CA, USA, 8-13 May 1994; pp. 781-786.

17. Chaker, A.; Mlika, A.; Laribi, M.A.; Romdhane, L.; Zeghloul, S. Synthesis of spherical parallel manipulator for dexterous medical task. Front. Mech. Eng. 2012, 7, 150-162. [CrossRef]

18. Saafi, H.; Laribi, M.A.; Zeghloul, S. Resolution of Spherical Parallel Manipulator (SPM) forward Kinematic Model (FKM) Near the Singularity. In Proceedings of the 2013 21ème Congrès Français de Mécanique, Bordeaux, 26-30 August 2013.

19. Houssem, S.; Laribi, M.A.; Zeghloul, S. Forward Kinematic Model Resolution of a Special Spherical Parallel Manipulator: Comparison and Real-Time Validation. Robotics 2020, 9, 62. [CrossRef]

20. Sadeqi, S.; Bourgeois, S.P.; Park, E.J.; Arzanpour, S. Design and performance analysis of a 3-RRR spherical parallel manipulator for hip exoskeleton applications. J. Rehabil. Assist. Technol. Eng. 2017, 4, 2055668317697596. [CrossRef] [PubMed]

21. Gao, J.S.; Wang, Y.C.; Liu, D.P.; Zuo, W.L. Simulation and experiment on the serial-parallel leg of a novel quadruped walking robot. In Proceedings of the 2016 IEEE International Conference on Mechatronics and Automation, Harbin, China, 7-10 August 2016; pp. 165-170. [CrossRef]

22. Hofschulte, J. Zweibeiniger Roboter Mit Parallelkinematischen Hüftgelenken; Books on Demand GmbH: Norderstedt, Germany, 2006. 
23. Wu, G.; Bai, S.; Kepler, J. Mobile platform centre shift in spherical parallel manipulators with flexible limbs. Mech. Mach. Theory 2014, 75, 12-26. [CrossRef]

24. Al-Widyan, K.; Ma, X.Q.; Angeles, J. The robust design of parallel spherical robots. Mech. Mach. Theory 2011, 46, 335-343. [CrossRef]

25. Tsai, L.W. Robot Analysis: The Mechanics of Serial and Parallel Manipulators; Wiley-Interscience, John Wiley \& Sons, Inc.: Hoboken, NJ, USA, 1999.

26. Craig, J.J. Introduction to Robotics: Mechanics and Control, 3rd ed.; Pearson: London, UK, 2003.

27. Siciliano, B.; Khatib, O. Introduction. In Springer Handbook of Robotics; Siciliano, B., Khatib, O., Eds.; Springer: Berlin/Heidelberg, Germany, 2008. [CrossRef]

28. Formlabs: High resulution SLA and SLS 3D Printers for Professionals. Available online: https://formlabs.com/ (accessed on 10 March 2021).

29. CeramTec-The Ceramic Experts. 2020. Available online: https://www.ceramtec.de/ (accessed on 10 August 2020).

30. Robotis e-Manual XC430-W240-T. Available online: https://emanual.robotis.com/docs/en/dxl/x/xc430-w240/ (accessed on 10 January 2021).

31. Matlab/Simulink (documentation). Available online: https://www.mathworks.com/ (accessed on 1 August 2021).

32. GitHub ByteCake. Available online: https:/ / github.com/bytecake (accessed on 10 January 2021).

33. Flores, P.; Lankarani, H.M. Contact Force Models for Multibody Dynamics; Springer: Berlin/Heidelberg, Germany, 2016 ; Volume 226.

34. Silva, M.; Barbosa, R.; Castro, T. Multi-Legged Walking Robot Modelling in MATLAB/SimmechanicsTM and its Simulation. In Proceedings of the 2013 8th EUROSIM Congress on Modelling and Simulation, Cardiff, UK, 10-13 September 2013. [CrossRef]

35. BIOLOX Forte. Available online: https://www.ceramtec.com/biolox/materials/biolox-forte/ (accessed on 10 August 2020). 